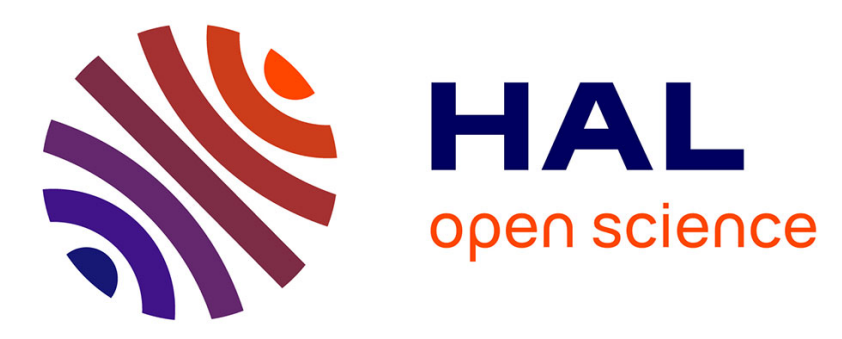

\title{
Numerical simulations of a transient injection flow at low Mach number regime
}

Alberto Beccantini, Etienne Studer, S. Gounand, J.-P. Magnaud, Thibaud Kloczko, Christophe Eric Corre, Sergueï Kudriakov

\section{To cite this version:}

Alberto Beccantini, Etienne Studer, S. Gounand, J.-P. Magnaud, Thibaud Kloczko, et al.. Numerical simulations of a transient injection flow at low Mach number regime. International Journal for Numerical Methods in Engineering, 2008, 76 (5), pp.662-696. 10.1002/nme.2331 . hal-00357645

\section{HAL Id: hal-00357645 https://hal.science/hal-00357645}

Submitted on 14 Jun 2021

HAL is a multi-disciplinary open access archive for the deposit and dissemination of scientific research documents, whether they are published or not. The documents may come from teaching and research institutions in France or abroad, or from public or private research centers.
L'archive ouverte pluridisciplinaire HAL, est destinée au dépôt et à la diffusion de documents scientifiques de niveau recherche, publiés ou non, émanant des établissements d'enseignement et de recherche français ou étrangers, des laboratoires publics ou privés. 
INTERNATIONAL JOURNAL FOR NUMERICAL METHODS IN ENGINEERING

Int. J. Numer. Meth. Engng 2000; 00:1-6 Prepared using nmeauth.cls [Version: 2002/09/18 v2.02]

\title{
Numerical simulations of a transient injection flow at low Mach number regime
}

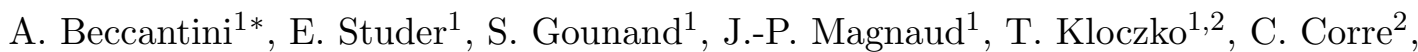 \\ S. Kudriakov ${ }^{1}$ \\ 1 CEA Saclay, DM2S/SFME/LTMF, 91191 Gif sur Yvette CEDEX, France \\ 2 SINUMEF Lab., ENSAM, 151 Bd de l'Hôpital, 75013 Paris, France
}

\section{SUMMARY}

In this paper, a transient injection flow at low Mach number regime is investigated. Three different methods are used and analyzed. Two of them are based on asymptotic models of the Navier-Stokes equations valid for small Mach numbers, whereas another is based on the full compressible NavierStokes equations, with particular care given to the discretization at low Mach numbers. Numerical solutions are computed both with or without the gravity force. Finally, the performance of the solvers in terms of CPU-time consumption is investigated and the sensitivity of the solution to some parameters which affect CPU-time is also performed. Copyright (c) 2000 John Wiley \& Sons, Ltd.

KEY WORDS: finite element method; finite volume method, BDF2, low Mach number flows, injection, laminar flow

\section{INTRODUCTION}

In nuclear engineering applications, Computational Fluid Dynamics (CFD) codes are increasingly becoming an important tool for design and analysis problems. For instance, CFD tools have been widely used for safety issues in the analysis of hypothetical loss-of-coolant accidents in Pressurized Light Water Reactor (see for instance [11, 25, 30]). CFD codes are also used for the design and analysis of systems for the next generation nuclear power plants, including the so-called High Temperature Gas Reactors [7].

According to $[9,22]$, when the temperature variations are large, the Boussinesq incompressible model cannot be used even if the Mach number is extremely low. In this case, low Mach number models can be used to take into account the compressibility effects due to the large variation of temperature. To assess the capabilities of CFD codes in computing low Mach number flows, a thorough validation process is needed, which includes calculation of experiments [29] as well as of analytical test cases. A reference test case for steady low Mach number flows is

${ }^{*}$ Correspondence to: A. Beccantini, CEA Saclay, DM2S/SFME/LTMF, Bat. 454, Point Courrier 47, 91191 Gif sur Yvette CEDEX, France. e-mail: beccantini@cea.fr 
the modeling and the computation of the natural convection in a square cavity with large temperature differences, presented in $[5,13,20,21]$ and which has been the object of an international workshop [15]. The purpose of our article is to provide an analytical test case for transient low Mach number flows and, more precisely, for injection problems in the low Mach number regime. Moreover, in this work, the behavior of different models in computing this kind of problems is also investigated.

As explained in [17], the main difficulty in constructing numerical methods for low Mach number flows is the fact that, in the transition from compressible to incompressible flows, the governing equations change nature: the Euler equations for compressible flows are hyperbolic and become hyperbolic-elliptic as the characteristic flow speed becomes zero compared with the sound speed.

There exists two families of solvers to compute flows at low Mach number regime.

The so-called density-based methods have widely been used to compute supersonic and transonic flows. Here the pressure is linked to the density and the internal energy via an equation of state. It is well-know that they cannot be used to compute flows at low-Mach regime without modification. The reason is the existence of a large disparity between the eigenvalues of the Euler equation which, as described in [35], reduces the accuracy of the solver. In the last decade, different techniques have been developed to extend these solvers to the quasi-incompressible regime [33], based on the modification of the time dependent properties of the governing equations (to cluster the eigenvalues of the Euler equations) or by only modifying the numerical viscosity of the scheme, as in [35].

The so-called pressure-based methods have been widely used to compute incompressible flows. Here the pressure variations are independent from the equation of state and are coupled to the divergence condition on the velocity. Pressure is always computed implicitly, for example as the solution of a Poisson-like equation. It is well-known that the Boussinesq incompressible model cannot be used if the temperature variations are large even if the Mach number is extremely low [9]. Then, when the temperature variations are too high and/or when the characteristic Mach number is not negligible, pressure-based methods which solves low Mach number equations arising from the asymptotics of the compressible equations have been developed. In [17] it is stated that these methods are more robust than density-based solvers. Nevertheless, the range of validity of pressure-based solvers arising from asymptotics is in general more limited than the one of density-based solver (which can be developed to compute flows at all speeds).

This paper is divided into several sections. In section 2 we describe the injection test case. As in the benchmark of the square cavity with large temperature differences $[13,21,20]$, we restrict our attention to a single-component, calorically perfect gas and laminar flow. As already mentioned, in the benchmark of the square cavity with large temperature differences only stationary test cases are investigated; the injection problem considered here is time-dependent. The description of fully compressible equations and the ones arising from asymptotic analysis with respect to the Mach number is the object of section 3 (some details concerning asymptotic analysis of the compressible equations are presented in appendix 9). In sections 4 and 5 we present the density-based and pressure-based solvers we use to compute the injection problem. All of them use unstructured grids in order to be able to investigate complex geometries. Nevertheless, the numerical results presented here have been obtained on Cartesian regular grids to enhance their accuracy. In section 6 , we present the numerical solution of the problem. Finally, in section 7, we investigate the performance of the solvers in terms of CPU cost and 


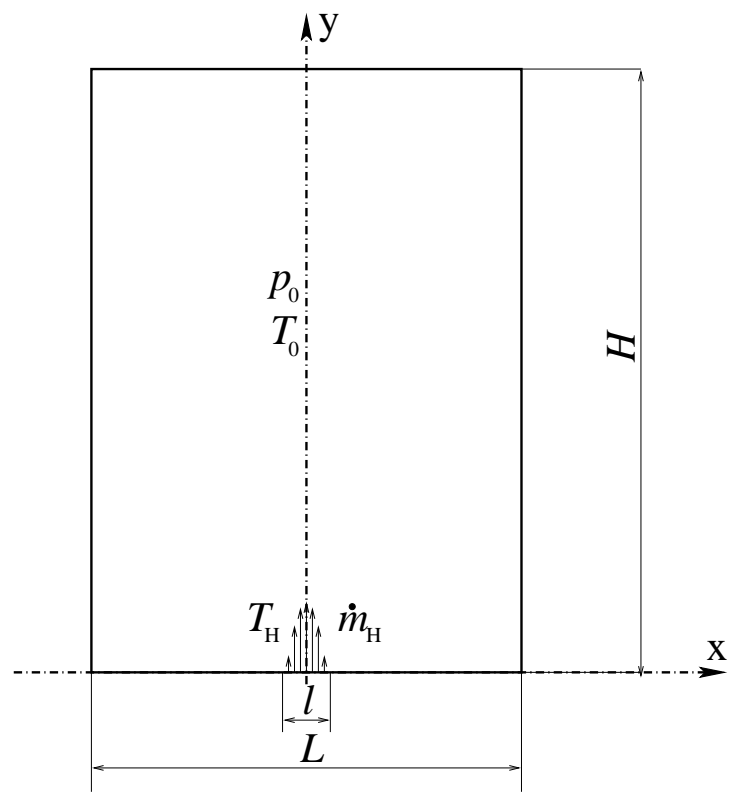

Figure 1. 2D planar rectangular cavity initially filled with a gas at rest. Hot gas is injected through the opening at the bottom.

accuracy of the solution; we also perform a sensitivity analysis of the solution with respect to some numerical parameters, such as the CFL or the convergence error in the fixed-point iteration, which allow us to reduce the CPU time. As we show, the larger the CFL, the lower the CPU time; the larger the convergence error in the fixed-point iterations, the lower the CPU time. Unfortunately, the accuracy is strongly affected. Conclusion follows (section 8).

\section{THE INJECTION TEST-CASE}

We consider the 2D planar rectangular cavity of figure 1 . We suppose that it is initially filled with a calorically perfect gas, with constant temperature $T_{0}$ and constant pressure $p_{0}$. We inject the same gas from the bottom part of the cavity ( $l$ is the opening width). The cavity walls are assumed to be impermeable and adiabatic. The injected flow temperature is $T_{H}$; the momentum shape at the inlet is parabolic and symmetric with respect to the $y$-axis; it is zero on the cavity walls (non-slip boundary conditions).

The dimensions of the cavity are

$$
\begin{aligned}
& L=3 \mathrm{~m}, \\
& H=7 \mathrm{~m},
\end{aligned}
$$

while the dimension of the injection opening is

$$
l=0.2 \mathrm{~m} .
$$


The gas specific heat ratio and gas constant are

$$
\begin{array}{ccc}
\gamma & = & 1.4 \\
R & = & 287 \mathrm{~J} \mathrm{~kg}^{-1} \mathrm{~K}^{-1}
\end{array}
$$

respectively.

At the beginning the gas in the cavity is at rest, with pressure, temperature and density given by

$$
\begin{aligned}
p_{0} & =10^{5} \mathrm{~Pa}, \\
T_{0} & =300 \mathrm{~K}, \\
\rho_{0} & =\frac{p_{0}}{R T_{0}} .
\end{aligned}
$$

The injection boundary conditions are given by an average momentum and a temperature

$$
\begin{aligned}
\dot{m}_{H} & =1.0 \mathrm{~kg} / \mathrm{m}^{2} / \mathrm{s} \\
T_{H} & =600 \mathrm{~K} ;
\end{aligned}
$$

The parabolic profile of the momentum, parallel to the $y$-axis is

$$
\rho u_{y}=\frac{6 \dot{m}_{H}}{l^{2}}\left(\frac{l^{2}}{4}-x^{2}\right) .
$$

The reason for specifying such boundary conditions on the mass flow and the temperature is that, when injection experiments are performed, these are the quantities that we know when the injection is realized using a high pressure reservoir coupled with a de Laval nozzle. In low Mach number flows, the temperature at the injection is equal to reservoir temperature (the total enthalpy being constant and the kinetic energy at the injection being negligible), and the average mass flow is constant in time (insensitive to the value of the internal pressure) and given by the sonic condition at the throat.

Note that, with the given numerical values, the flow is subsonic and in a low Mach number regime. Indeed, the order of magnitude of the flow velocity in the vessel is the same as in the injection region:

$$
u_{H} \approx \frac{\dot{m}_{H}}{\rho_{H}} \approx \frac{\dot{m}_{H}}{p_{0}} R T_{H} \approx 1.7 \mathrm{~m} / \mathrm{s} .
$$

The sound speed is initially about $\sqrt{\gamma R T_{0}}=350 \mathrm{~m} / \mathrm{s}$ in the cavity and $500 \mathrm{~m} / \mathrm{s}$ at the injection, i.e. much larger than the flow velocity. Then we can define a reference Mach number

$$
\mathrm{M}=\frac{\dot{m}_{H}}{p_{0}} R T_{H} \cdot \frac{1}{\sqrt{\gamma R T_{0}}} \approx 4.86 \cdot 10^{-3} .
$$

For the sake of simplicity, we suppose that the dynamic viscosity and thermal diffusivity are constant. We define them by introducing reference Reynolds and Prandtl numbers

$$
\begin{gathered}
\operatorname{Re}=\frac{\dot{m}_{H} l}{\mu}, \\
\operatorname{Pr}=\frac{\mu}{\lambda} c_{p}=\frac{\mu}{\lambda} \frac{\gamma}{\gamma-1} R,
\end{gathered}
$$


which imply that the dynamic viscosity and the thermal conductivity are given by

$$
\begin{gathered}
\mu=\frac{\dot{m}_{H} l}{\operatorname{Re}}, \\
\lambda=\frac{\dot{m}_{H} l}{\operatorname{Re} \operatorname{Pr}} \frac{\gamma}{\gamma-1} R .
\end{gathered}
$$

In this work, we restrict our attention to two different test cases:

C1. gravity $g=0, \operatorname{Re}=40$., $\operatorname{Pr}=0.71$;

C2. gravity $g=9.81 \mathrm{~m} \mathrm{~s}^{-2} \operatorname{Re}=40$., $\operatorname{Pr}=0.71$;

namely we consider the same (laminar) case and we neglect or not the gravity force. In the latter case the value of the Froude number is

$$
\mathrm{Fr}=\frac{u_{H}^{2}}{g H} \approx 0.042,
$$

i.e. we expect that the gravity drastically changes the flow obtained in the case $\mathrm{C} 1$.

In both cases we represent the numerical results at $t=6 \mathrm{~s}$. At this time, as we will see, in the former case the entering gas only occupies a region closed to the injection; in the latter case, because of buoyancy effects, the entering gas reaches the top of the cavity.

\subsection{Conservation properties}

2.1.1. Mass conservation. According to the boundary conditions, the total mass in the cavity can be computed as a function of time:

$$
\int_{V} \rho(\vec{r}, t) \mathrm{d} V=\int_{V} \rho(\vec{r}, 0) \mathrm{d} V+\dot{m}_{H} l t
$$

2.1.2. Energy conservation. At low Mach number the kinetic energy is small compared to the internal energy (see appendix 9); then we can write that the total energy

$$
\rho e_{t} \approx \frac{1}{\gamma-1} p
$$

The total energy flux into the cavity is given by

$$
\dot{m}_{H} \frac{\gamma}{\gamma-1} R T_{H}+\dot{m}_{H} 0.5 \vec{u} \cdot \vec{u}-\lambda \frac{\partial T}{\partial y} \approx \dot{m}_{H} \frac{\gamma}{\gamma-1} R T_{H}\left(1-\frac{l}{T_{H} \operatorname{Re} \operatorname{Pr}} \frac{\partial T}{\partial y}\right) .
$$

If we neglect the contribution of the diffusive term, the average pressure can be computed as

$$
\int_{V} p(\vec{r}, t) \mathrm{d} V \approx \int_{V} p(\vec{r}, 0) \mathrm{d} V+\gamma \dot{m}_{H} R T_{H} l t .
$$




\subsection{Entropy behavior.}

The injection of hot gas in the cavity causes the compression of the cold gas inside. From a physical point of view, this is due to the acoustic waves, which travel into the cavity to establish a mechanical equilibrium, i.e. constant thermodynamic pressure. Because of this compression, even in the region far from the injection and at the initial time (i.e. before that the entering gas reaches this region), the temperature instantaneously increases. Indeed,

$$
\begin{aligned}
T \mathrm{~d} s & =\mathrm{d} h-\frac{1}{\rho} \mathrm{d} p \\
& =\frac{\gamma R}{\gamma-1} \mathrm{~d} T-\frac{R T}{p} \mathrm{~d} p .
\end{aligned}
$$

Far from the injection, the flow is initially subjected to an adiabatic and isentropic compression only. Then, in this region, we have $T \mathrm{~d} s=0$, i.e.

$$
\frac{\gamma}{\gamma-1} \frac{\mathrm{d} T}{T}=\frac{\mathrm{d} p}{p},
$$

which gives

$$
T=T_{0}\left(\frac{p}{p_{0}}\right)^{\frac{\gamma-1}{\gamma}} .
$$

\section{GOVERNING EQUATIONS}

We consider the Navier-Stokes equations for a compressible, calorically perfect gas, namely

$$
\left\{\begin{array}{l}
\frac{\partial \rho}{\partial t}+\vec{\nabla} \cdot(\rho \vec{u})=0 \\
\frac{\partial \rho \vec{u}}{\partial t}+\vec{\nabla} \cdot(\rho \vec{u} \otimes \vec{u}+p \underline{I})=\rho \vec{g}+(\vec{\nabla} \cdot \underline{\tau}) \\
\frac{\partial \rho e_{t}}{\partial t}+\vec{\nabla} \cdot\left(\rho \vec{u} h_{t}\right)=\vec{\nabla} \cdot(\lambda \vec{\nabla} T)+\rho \vec{g} \cdot \vec{u}+\vec{\nabla} \cdot(\underline{\tau} \cdot \vec{u}) \\
p=\rho R T=(\gamma-1) \rho\left(e_{t}-\frac{1}{2} \vec{u} \cdot \vec{u}\right)
\end{array}\right.
$$

where $\rho$ is the density, $\vec{u}$ the velocity, $p$ the pressure, $\vec{g}$ the gravity, $\underline{\tau}$ the viscosity stress tensor, defined by

$$
\underline{\tau}=\mu\left(\vec{\nabla} \otimes \vec{u}+(\vec{\nabla} \otimes \vec{u})^{T}-\frac{2}{3}(\vec{\nabla} \cdot \vec{u}) \underline{I}\right),
$$

$e_{t}$ the unit mass total (kinetic+internal) energy and $h_{t}$ the unit mass total enthalpy, $T$ the temperature, $\mu$ the dynamic viscosity and $\lambda$ the thermal conductivity. The first equation of (4) expresses the mass conservation, the second one the momentum conservation, the third one the energy conservation. The last one is the equation of state. 
In $[16,35]$, single time scale and single space scale asymptotic analysis for the Navier-Stokes equations at low-Mach number regime is presented, under the hypotheses

$$
\frac{\rho g L}{p}<<1
$$

and

$$
\frac{\mathrm{M}^{2}}{\mathrm{Re}}<<1
$$

where $\mathrm{M}$ is the reference Mach number. By inserting the asymptotic expansions of the variables with respect to the reference Mach number in the fully-compressible Navier-Stokes equations (see appendix 9 for details), it can be shown that the pressure can be split in two terms, i.e.

$$
p(\vec{r}, t)=P(t)+p^{\prime}(\vec{r}, t),
$$

in which $P(t)$ is the so-called (constant in space) thermodynamic pressure $(P=O(1)$ with respect to the reference Mach number) and $p^{\prime}(\vec{r}, t)$ is the so-called dynamic pressure $\left(p^{\prime}=O\left(\mathrm{M}^{2}\right)\right)$. According to single time scale and single space scale asymptotics, in the particular case of a calorically perfect gas, we have to find $P(t), \vec{u}(\vec{r}, t), T(\vec{r}, t)$ and $p^{\prime \prime}(\vec{r}, t)$, solutions of the initial value problem

$$
\left\{\begin{array}{l}
\frac{1}{\gamma P} \frac{\mathrm{d} P}{\mathrm{~d} t}+(\vec{\nabla} \cdot \vec{u})=\frac{\gamma-1}{\gamma P} \vec{\nabla} \cdot(\lambda \vec{\nabla} T) \\
\frac{\partial T}{\partial t}+(\vec{u} \cdot \vec{\nabla}) T=\frac{\gamma-1}{\gamma} \frac{T}{P}\left(\frac{\mathrm{d} P}{\mathrm{~d} t}+\vec{\nabla} \cdot(\lambda \vec{\nabla} T)\right) \\
\frac{\partial \vec{u}}{\partial t}+(\vec{u} \cdot \vec{\nabla}) \vec{u}=\frac{R T}{P}\left(-\vec{\nabla} p^{\prime \prime}+\vec{\nabla} \cdot \underline{\tau}\right)+\vec{g}\left(1-\frac{T P_{0}}{T_{0} P}\right)
\end{array}\right.
$$

where $p^{\prime \prime}=p^{\prime}+\rho_{0} g y$.

The first equation of (6) is the equation of conservation of internal energy (which is equal to zero-th order total energy with respect to M). It can be written in the form

$$
\vec{\nabla} \cdot \vec{u}=-\frac{1}{\gamma P} \frac{\mathrm{d} P}{\mathrm{~d} t}+\frac{\gamma-1}{\gamma P} \vec{\nabla} \cdot(\lambda \vec{\nabla} T) .
$$

The first term appearing in the RHS of (7) is constant (in space) and is linked to the isentropic compression of the fluid elements due to infinitely fast acoustic waves. The second term expresses the variation of the velocity divergence linked to the temperature distribution in space. The second equation of (6) is a time-evolution equation for the temperature. Here we can see three terms which represent the three mechanisms which are responsible for the temperature variation: the convection term, the diffusion term and the compression (which produces temporal variation of the thermodynamic pressure everywhere because of the infinitely fast acoustic waves). We also note that the temperature and the velocity are linked by the coefficient appearing in the velocity equation but also via the divergence condition (7).

As far as the computation of $P(t)$ is concerned, different approaches are possible [8]. Integrating the first equation of $(6)$ over the volume of the cavity $(V=L H)$, we obtain, in our case,

$$
\frac{1}{\gamma-1} \frac{\mathrm{d} P}{\mathrm{~d} t} L H+\frac{\gamma}{\gamma-1} R T_{H} \dot{m}_{H} l=-\int_{\partial \Omega_{\mathrm{inj}}} \lambda \frac{\partial T}{\partial y} \mathrm{~d} S,
$$


where the last integral involves the injection surface. Equation (8) expresses the internal energy conservation over the total volume.

If we integrate the equation of state over the volume, we obtain

$$
\int_{\Omega} \rho \mathrm{d} V=\frac{P}{R} \int_{\Omega} \frac{1}{T} \mathrm{~d} V,
$$

i.e. in this particular case

$$
\frac{P}{R} \int_{\Omega} \frac{1}{T} \mathrm{~d} V=\rho_{0} L H+\dot{m}_{H} l t .
$$

Equation (10) expresses mass conservation in the cavity.

Then we can compute the thermodynamic pressure by imposing the conservation of energy (equation (8)) or the conservation of mass (equation (10)). Nevertheless in stationary problems, such as the previously mentioned problem of the natural convection in a square cavity $[13,20,21]$, the use of conservation of energy to determine the thermodynamic pressure prevents the algorithm from converging in time, since the temperature is not computed via a conservative equation and the integral of the heat flux over the surface is affected by the spatial numerical errors and is not zero. For this reason, in this work we have preferred to compute the thermodynamic pressure by imposing the conservation of mass (equation (10)).

The initial problem (6) can be also formulated as follows (see appendix 9 for details). We have to determine $P(t), \vec{u}(\vec{r}, t), T(\vec{r}, t), p^{\prime \prime}(\vec{r}, t)$ and $\rho(\vec{r}, t)$ such that

$$
\left\{\begin{array}{l}
\vec{\nabla} \cdot \vec{u}=-\frac{1}{\rho}\left(\frac{\partial \rho}{\partial t}+\vec{u} \cdot \vec{\nabla} \rho\right) \\
\rho \frac{\partial \vec{u}}{\partial t}+\rho \vec{u} \cdot \vec{\nabla} \vec{u}=-\vec{\nabla} p^{\prime \prime}+\vec{\nabla} \cdot \underline{\tau}+\left(\rho-\rho_{0}\right) \vec{g} \\
\rho c_{p} \frac{\partial T}{\partial t}+\rho c_{p} \vec{u} \cdot \vec{\nabla} T=\vec{\nabla} \cdot(\lambda \vec{\nabla} T)+\frac{\mathrm{d} P}{\mathrm{~d} t}
\end{array}\right.
$$

$c_{p}$ being the specific heat at constant pressure (in this case $c_{p}=\gamma R /(\gamma-1)$ ).

As in the case of the system of equations (6), the thermodynamic pressure $P(t)$ is recovered by imposing the conservation of mass (equation (10)).

The density $\rho(\vec{r}, t)$ is recovered from the equation of state which, in the particular case of a calorically perfect gas, reads

$$
\rho(\vec{r}, t)=\frac{P(t)}{R T(\vec{r}, t)} .
$$

By comparing the systems of equations (6) and (11), we can say that:

- In the former system only 4 variables have to be computed while in the latter the variables are 5 .

- As we will show, the first equation of the systems (6) and (11) are used as a divergence condition. In a Galerkin approach, they are projected over the space of the test (and shape) functions of the dynamic pressure. Since in the system (6) second order derivatives are involved, it is necessary that these test functions are at least continuous. Conversely, in system (11) discontinuous shape functions for the pressure can be used.

- The approach used for (11) can be extended in a straightforward manner to other cases in which the gas is not calorically perfect and the equation of state is different from (12). 
In conclusion, if we use continuous shape and test functions for the pressure, the solution of the system of equations (6) is easier. Nevertheless, since the extension to gases with a general equation of state is easier for (11), we have developed two numerical approaches, one based on the former system, one on the latter.

\section{THE DENSITY-BASED SOLVER}

The Navier-Stokes equations (4) can be written in compact form as

$$
P_{c}^{-1} \frac{\partial w}{\partial \tau}+\frac{\partial w}{\partial t}+\vec{\nabla} \cdot\left(f^{E}-f^{V}\right)=S
$$

where $w$ is the "vector" of the conserved variable $\left(\rho, \rho \vec{u}, \rho e_{t}\right)^{T}, \tau$ a pseudo or dual-time, $t$ the physical time, $f^{E}$ and $f^{V}$ are respectively the convective flux and viscous flux (E standing for Euler, $\mathrm{V}$ for viscous), $\mathrm{S}$ is the source term involving $\vec{g}, P_{c}$ is a preconditioning matrix which takes, in the present work, the form proposed in [32].

In the density-based solver (or fully-compressible solver), we solve the conservative equations (13) using unstructured Finite Volume approach for space discretization and BDF2 (second order accurate Backward Difference Formula) for the real time discretization and the Euler implicit scheme (or BDF1) for the "preconditioned" dual-time. Implicit schemes are necessary if we want to use time steps larger than the ones based on the CFL condition, which involves the acoustic wave speed. The numerical scheme thus obtained can be written as

$$
\begin{aligned}
\left(P_{c}^{-1}\right)_{i}^{n, \ell} \frac{\Delta w_{i}^{n, \ell}}{\Delta \tau_{i}^{n, \ell}} & +\frac{\frac{3}{2}\left(w_{i}^{n, \ell}-w_{i}^{n}\right)-\frac{1}{2} \Delta w_{i}^{n-1}}{\Delta t} \\
& +\frac{1}{\Omega_{i}} \sum_{k}\left(\mathcal{H}_{i, k}^{E}-\mathcal{H}_{i, k}^{V}\right)^{n, \ell+1} \cdot \vec{n}_{i, k} S_{i, k}=\mathcal{S}_{i}^{n, \ell+1},
\end{aligned}
$$

where $\ell$ is the pseudo-iteration (on dual-time) counter, $n$ is the time step counter, $\Delta w^{n, \ell}=$ $w^{n, \ell+1}-w^{n, \ell}, \Delta w^{n-1}=w^{n}-w^{n-1}, i$ is the cell counter, $k$ is the counter for the face center (index " $i, k$ " represents the center of the $k$-th interface of the $i$-th cell), $\Omega_{i}$ is the volume of the $i$-th cell, $\mathcal{H}^{E}$ (respectively $\mathcal{H}^{V}$ ) is the numerical approximation of the non-viscous flux (respectively viscous flux), $\mathcal{S}$ is the numerical approximation of the gravity terms, $\vec{n}$ is the outward normal, $S$ is the surface. Scheme (14) can be expressed also written as

$$
\left(P_{c}^{-1}\right)_{i}^{n, \ell} \frac{\Delta w_{i}^{n, \ell}}{\Delta \tau_{i}^{n, \ell}}+\frac{3}{2} \frac{\Delta w_{i}^{n, \ell}}{\Delta t}+\frac{1}{\Omega_{i}} \sum_{k}\left(\Delta \mathcal{H}_{i, k}\right)^{n, \ell} \cdot \vec{n}_{i, k} S_{i, k}-\left(\Delta \mathcal{S}_{i}\right)^{n, \ell}=-(\mathcal{R})_{i}^{n, \ell},
$$

where the explicit residuum is given by

$$
(\mathcal{R})_{i}^{n, \ell}=\frac{1}{\Omega_{i}} \sum_{k}\left(\mathcal{H}_{i, k}\right)^{n, \ell} \cdot \vec{n}_{i, k} S_{i, k}+\frac{\frac{3}{2}\left(w_{i}^{n, \ell}-w_{i}^{n}\right)-\frac{1}{2} \Delta w_{i}^{n-1}}{\Delta t}-\left(\mathcal{S}_{i}\right)^{n, \ell}
$$

and $\mathcal{H}=\mathcal{H}^{E}-\mathcal{H}^{V}$.

As one can see, once the convergence on the counter $\ell$ (i.e. the steady state for $\tau$ ) is reached, namely

$$
\lim _{\ell \rightarrow \infty} \Delta w^{n, \ell}=0,
$$


the LHS of equation (15) goes to 0. Then, the numerical approach used to compute the LHS of equation (15) does not affect the (space and time) accuracy of the method (providing that the convergence on $\ell$ is reached) but only the convergence speed. For this reason, the numerical schemes used to compute the LHS (15) can be simpler to deal with than the ones used to compute the RHS. In this case, it is said that the numerical approach used to compute the LHS of (15) is not consistent with the one used in the RHS [23].

As far as the RHS of (15) is concerned, inviscid fluxes are computed using the HLLC scheme of Toro [31], extended to (theoretical) second order using the primitive variable reconstruction of Barth and Jespersen, described in [1] and belonging to the MUSCL family; moreover the numerical dissipation of the inviscid scheme is corrected taking into account Low Mach number preconditioning [14]. Viscous fluxes are approximated using a linearly-exact extension of the diamond method of Noh [19].

Concerning the inviscid flux for the LHS of (15), we linearize (around $w^{n, \ell}$ ) the Rusanov first-order accurate scheme (in which the numerical dissipation is corrected taking into account low Mach number preconditioning). Conversely the diffusive flux and source term have been exactly linearized. Then, the computation of the state $\Delta w^{n, \ell+1}$ is performed using GMRES(50) (restart performed each 50-th iteration) coupled with the ILUTP preconditioner (the dimension of which is taken 1.5 times larger than the matrix involved in the linear system) [26, 27]. This algorithm is implemented in the CEA code CAST3M (described in [4]).

\section{THE PRESSURE-BASED SOLVERS}

\subsection{Time discretization}

As already mentioned, time discretization is performed via a Finite Difference approach. For the sake of simplicity, let us consider the backward Euler implicit formula (BDF1); the extension to BDF2 is straightforward.

Asymptotic approach 1. Let us consider the initial value problem (6). This problem is nonlinear. In order to treat the non-linearities, we use a fixed-point approach. We suppose that, at $t=t^{n}$, we know the variables $P^{n}, T^{n}, \vec{u}^{n}, p^{\prime \prime}{ }^{n}$. Let us describe how to compute $P^{n+1}, T^{n+1}$, $\vec{u}^{n+1}, p^{\prime \prime}{ }^{n+1}$.

In the following, we let $\ell$ denote the index referring to the fixed-point iteration. Note that, for $\ell=0$, the generic variable $a^{n+1, \ell}$ is equal to $a^{n}$.

At the $\ell$-th fixed-point iteration, we first compute the pressure via equation (10):

$$
\frac{P^{n+1, \ell+1}}{R} \int_{\Omega} \frac{1}{T^{n+1, \ell}} \mathrm{d} V=\rho_{0} L H+\dot{m}_{H} l t^{n+1} .
$$

Then we compute the average velocity at the injection. Indeed, at the injection boundary, the average velocity is

$$
\frac{\dot{m}_{H}}{\rho^{n+1, \ell+1}}=\frac{\dot{m}_{H}}{P^{n+1, \ell+1}} R T_{H} .
$$

Then, at the injection boundary, the velocity is given by

$$
u_{y}^{n+1, \ell+1}=\frac{6 \dot{m}_{H} R T_{H}}{P^{n+1, \ell+1}}\left(\frac{1}{4}-\frac{x^{2}}{l^{2}}\right) .
$$

Copyright (C) 2000 John Wiley \& Sons, Ltd.

Int. J. Numer. Meth. Engng 2000; 00:1-6

Prepared using nmeauth.cls 
Now, using the compatibility condition arising from $(7)^{\dagger}$

$$
\frac{1}{\gamma P^{n+1, \ell+1}}\left(\frac{\mathrm{d} P}{\mathrm{~d} t}\right)^{n+1, \ell+1} L H+\frac{\dot{m}_{H} R T_{H}}{P^{n+1, \ell+1}} l=\frac{\gamma-1}{\gamma P^{n+1, \ell+1}} \int_{\partial \Omega} \vec{\nabla} \cdot\left(\lambda \vec{\nabla} T^{n+1, \ell}\right) \mathrm{d} S,
$$

we evaluate the time derivative of the pressure in the divergence condition.

Then the condition on the divergence of $\vec{u}^{n+1}$ is given by

$$
\frac{1}{\gamma P^{n+1, \ell+1}}\left(\frac{\mathrm{d} P}{\mathrm{~d} t}\right)^{n+1, \ell+1}+\left(\vec{\nabla} \cdot \vec{u}^{n+1, \ell+1}\right)=\frac{\gamma-1}{\gamma P^{n+1, \ell+1}} \vec{\nabla} \cdot\left(\lambda \vec{\nabla} T^{n+1, \ell}\right) .
$$

Note that, the velocity field $\vec{u}^{n+1, \ell}$, used in the non-linear convective term in a fixed-point strategy, does not satisfy the compatibility condition for the velocity (which varies with $\ell$ ) until the convergence of the fixed-point is reached. In order to enforce this condition for each $\ell$, we correct the velocity $\vec{u}^{n+1, \ell}$ on the injection by imposing the condition (17). From a practical point of view, this correction enhances the convergence speed of the fixed-point strategy.

Let us evaluate the temperature $T^{n+1, \ell+1}$ :

$$
\begin{aligned}
\left(\frac{T^{n+1, \ell+1}-T^{n}}{\Delta t}\right)+\left(\vec{u}^{n+1, \ell} \cdot \vec{\nabla}\right) T^{n+1, \ell+1} & =\frac{\gamma-1}{\gamma} \frac{T^{n+1, \ell}}{P^{n+1, \ell+1}}\left(\frac{P^{n+1, \ell+1}-P^{n}}{\Delta t}\right. \\
& \left.+\vec{\nabla} \cdot\left(\lambda \vec{\nabla} T^{n+1, \ell+1}\right)\right) .
\end{aligned}
$$

Finally, we have to compute the velocity $\vec{u}^{n+1}$ and the dynamic pressure $p^{\prime \prime}{ }^{n+1}$. We have used a "fully implicit approach", in which we solve

$$
\begin{aligned}
& \frac{\vec{u}^{n+1, \ell+1}-\vec{u}^{n}}{\Delta t}+\left(\vec{u}^{n+1, \ell} \cdot \vec{\nabla}\right) \vec{u}^{n+1, \ell+1}=\frac{R T^{n+1, \ell+1}}{P^{n+1, \ell+1}}\left(-\vec{\nabla} p^{\prime \prime}{ }^{n+1, \ell+1}+\vec{\nabla} \cdot \underline{\tau}^{n+1, \ell+1}\right) \\
& +\vec{g}\left(1-\frac{T^{n+1, \ell+1} P_{0}}{T_{0} P^{n+1, \ell+1}}\right),
\end{aligned}
$$

coupled to the divergence constraint (19).

Asymptotic approach 2. Let us consider the system of equation (11). We suppose that, at $t=t^{n}$, we know the variables $P^{n}, T^{n}, \vec{u}^{n}, p^{\prime \prime n}$ and the density $\rho^{n}$. Let us describe how to compute $P^{n+1}, T^{n+1}, \vec{u}^{n+1}, p^{\prime \prime n+1}$. The non-linearities are solved with a fixed point algorithm. At the $\ell$-th fixed-point iteration, we first compute the pressure via equation (9):

$$
P^{n+1, \ell+1} \int_{\Omega} \frac{1}{R T^{n+1, \ell}} \mathrm{d} V=\rho_{0} L H+\dot{m}_{H} l t^{n+1}
$$

\footnotetext{
${ }^{\dagger}$ This compatibility condition is obtained by integrating equation (7) over the volume. Here we enforce the discrete solution to satisfy such compatibility condition. 
Then we compute the injection velocity using (17), i.e.

$$
u_{y}^{n+1, \ell+1}=\frac{6 \dot{m}_{H} R T_{H}}{P^{n+1, \ell+1}}\left(\frac{1}{4}-\frac{x^{2}}{l^{2}}\right) .
$$

The condition on the divergence of $\vec{u}^{n+1}$ is given by:

$$
\vec{\nabla} \cdot \vec{u}^{n+1, \ell+1}=-\frac{1}{\rho^{n+1, \ell}}\left(\frac{\rho^{n+1, \ell}-\rho^{n}}{\Delta t}+\vec{u}^{n+1, \ell} \cdot \vec{\nabla} \rho^{n+1, \ell}\right)+B .
$$

The constant $B$ is introduced to exactly satisfy the compatibility condition

$$
\int_{\Omega}(\vec{\nabla} \cdot \vec{u}) \mathrm{d} V=\frac{\dot{m}_{H} R T_{H}}{P^{n+1, \ell+1}} l
$$

i.e.

$$
B=\frac{1}{V}\left[\frac{\dot{m}_{H} R T_{H} l}{P^{n+1, \ell+1}}+\int_{\Omega} \frac{1}{\rho^{n+1, \ell}}\left(\frac{\rho^{n+1, \ell}-\rho^{n}}{\Delta t}+\vec{u}^{n+1, \ell} \cdot \vec{\nabla} \rho^{n+1, \ell}\right) \mathrm{d} V\right] .
$$

Let us evaluate the velocity $\vec{u}^{n+1, \ell+1}$ and the pressure $p^{\prime \prime n+1, \ell+1}$ using a "fully implicit approach" ${ }^{\prime}$

$$
\begin{aligned}
& \rho^{n+1, \ell} \frac{\vec{u}^{n+1, \ell+1}-\vec{u}^{n}}{\Delta t}+\rho^{n+1, \ell}\left(\vec{u}^{n+1, \ell} \cdot \vec{\nabla}\right) \vec{u}^{n+1, \ell+1}= \\
& -\vec{\nabla} p^{\prime \prime n+1, \ell+1}+\vec{\nabla} \cdot \tau^{n+1, \ell+1}+\vec{g}\left(\rho^{n+1, \ell}-\rho_{0}\right) .
\end{aligned}
$$

The viscosity stress tensor $\underline{\tau}^{n+1, \ell+1}$ is evaluated using:

$$
\underline{\tau}^{n+1, \ell+1}=\mu\left(\vec{\nabla} \otimes \vec{u}^{n+1, \ell+1}+\left(\vec{\nabla} \otimes \vec{u}^{n+1, \ell+1}\right)^{T}-\frac{2}{3}\left(\vec{\nabla} \cdot \vec{u}^{n+1, \ell}\right) \underline{I}\right) .
$$

The temperature $T^{n+1, \ell+1}$ is evaluated via

$$
\begin{aligned}
& \rho^{n+1, \ell} \frac{T^{n+1, \ell+1}-T^{n}}{\Delta t}+\rho^{n+1, \ell}\left(\vec{u}^{n+1, \ell+1} \cdot \vec{\nabla}\right) T^{n+1, \ell+1}= \\
& \frac{1}{c_{p}}\left(\frac{\mathrm{d} P}{\mathrm{~d} t}\right)^{n+1, \ell+1}+\frac{1}{c_{p}} \vec{\nabla} \cdot\left(\lambda \vec{\nabla} T^{n+1, \ell+1}\right) .
\end{aligned}
$$

Finally the density $\rho^{n+1, \ell+1}$ is evaluated introducing into the Equation of State the values $P^{n+1, \ell+1}$ and $T^{n+1, \ell+1}$.

\subsection{Space discretization}

The space discretization of the equations is performed via the finite element solver available in the CAST3M code [4]. Several couples of finite elements are available to discretize the pressure/velocity couple in the Navier-Stokes equations.

$\ddagger$ Once again, "fully implicit" means that equation (24) is solved together with the divergence condition (23). 
Asymptotic approach 1. The velocity is approximated by using Lagrange complete quadratic polynomials (Q2). These polynomials involve linear combinations of $1, x, y, x^{2}, x y, y^{2}, x y^{2}$, $x^{2} y x^{2} y^{2}$ (in the frame of the reference element, see [6]). The pressure is approximated by using bilinear polynomials (Q1). These polynomials involve linear combinations of $1, x, y, x y$. The temperature is approximated by using Lagrange complete quadratic polynomials (as the velocity). We emphasize that the chosen couple of elements for the velocity and the pressure gives a LBB stable discretization for the Stokes problem [2] (with appropriate initial and boundary conditions).

Asymptotic approach 2. The velocity, the temperature (and the density) are approximated in the same manner as in the asymptotic approach 1 . The pressure is approximated by using linear non conforming polynomials (discontinuous at the interfaces of the elements). The chosen couple of elements (Q2 -P1 NC) for the velocity and the pressure gives a LBB stable discretization for the Stokes problem [2]. According to [10], page 464, for the incompressible Navier-Stokes equations the element "Q2 -P1 NC" is "probably the most accurate 2D element". Note also that, on a mesh with the same number of elements, the degrees of freedoms of the pressure are larger in this approach than in the asymptotic approach 1.

In both approaches, the solution of the linear systems arising from the linearization of the non-linear problem is obtained using GMRES(50) coupled with an ILUTP preconditioner.

\section{NUMERICAL RESULTS}

6.1. Case C1: $g=0$ and $R e=40$.

6.1.1. Solution analysis. Let us present the numerical results in the first case (no gravity) at $t=6 \mathrm{~s}$. These results have been obtained via the asymptotic approach 1 using a regular mesh of $120 \times 120$ elements and a reference CFL (Courant-Friedrich-Levy) number

$$
\mathrm{CFL}=\frac{\dot{m}_{H} \Delta t}{\rho_{0} \Delta x}=2
$$

The results obtained with the other approaches are very close to these ones (as we will show further in the grid convergence study).

In figure 2 we represent the velocity field, some streamtraces (lines which are tangent to the velocity field) and the temperature isocontours. In figure 3 we represent the divergence of $\vec{u}$. In figures 4 and 5 we show the evolution of $u_{x}, u_{y}$ and $T$ along some horizontal and vertical lines. The computed thermodynamic pressure is 1.139 bar, very close to the one given using formula (2) (1.138 bar), which does not take into account the heat flux at the injection due to thermal diffusion.

As one can see in figure 2 , the solution is symmetric with respect to the $y$-axis $(x=0)$. The inlet of flow in the cavity generates two vortices, which at $t=6 \mathrm{~s}$, are in $(x, y) \approx( \pm 0.45,2.2)$. Note that the streamtraces (lines which are tangent to the velocity field) are not closed lines. Indeed neither $\rho \vec{u}$ nor $\vec{u}$ are divergence free vectors; i.e. we cannot construct a streamline function whose isolines are tangent to the velocity vector.

As already mentioned in the analysis of the equation (7), we can decompose the velocity 
Table I. Case C1. Some values (SI units) along the axis $x=0$ at $t=6 \mathrm{~s}$ respectively given by the fully-compressible, the asymptotic 1 and the asymptotic 2 algorithms: grid convergence study. $\epsilon_{T}$ and $\epsilon_{\mathrm{u}_{\mathrm{y}}}$ are defined by equation (27).

\begin{tabular}{|c|c|c|c|c|c|c|c|c|}
\hline$y$ & Coarse & $\begin{array}{c}T \\
\text { Medium }\end{array}$ & Fine & $\begin{array}{c}\epsilon_{T}(\%) \\
\text { Fine }\end{array}$ & Coarse & $\begin{array}{c}u_{y} \cdot 100 \\
\text { Medium }\end{array}$ & Fine & $\begin{array}{c}\epsilon_{\mathrm{u}_{\mathrm{y}}}(\%) \\
\text { Fine }\end{array}$ \\
\hline \multirow[t]{3}{*}{0.5} & 557.11 & 560.06 & 561.45 & 0.6 & 167.67 & 165.92 & 164.93 & 0.3 \\
\hline & 564.92 & 565.17 & 565.00 & 0.07 & 164.46 & 163.90 & 164.29 & 0.1 \\
\hline & 567.23 & 565.25 & 564.96 & & 161.72 & 164.34 & 164.47 & \\
\hline \multirow[t]{3}{*}{1.0} & 520.69 & 522.82 & 524.02 & 0.6 & 137.46 & 135.33 & 134.14 & 0.4 \\
\hline & 526.87 & 527.48 & 527.24 & 0.02 & 132.69 & 133.45 & 133.48 & 0.08 \\
\hline & 528.79 & 527.49 & 527.14 & & 131.23 & 133.51 & 133.59 & \\
\hline \multirow[t]{3}{*}{1.5} & 497.34 & 499.80 & 500.90 & 0.7 & 122.26 & 120.68 & 120.52 & 0.9 \\
\hline & 504.12 & 504.76 & 504.48 & 0.02 & 119.80 & 119.35 & 119.37 & 0.07 \\
\hline & 505.45 & 504.69 & 504.36 & & 119.46 & 119.43 & 119.45 & \\
\hline \multirow[t]{3}{*}{2.0} & 476.77 & 478.78 & 479.55 & 0.7 & 126.72 & 127.69 & 128.49 & 0.3 \\
\hline & 483.63 & 483.62 & 483.15 & 0.04 & 128.82 & 128.70 & 128.83 & 0.05 \\
\hline & 484.17 & 483.37 & 482.95 & & 127.91 & 128.88 & 128.89 & \\
\hline \multirow[t]{3}{*}{2.5} & 462.77 & 465.04 & 466.80 & 0.6 & 114.53 & 110.94 & 106.64 & 0.1 \\
\hline & 471.51 & 470.57 & 469.95 & 0.08 & 105.27 & 106.43 & 106.36 & 0.1 \\
\hline & 472.75 & 470.21 & 469.57 & & 98.170 & 105.89 & 106.49 & \\
\hline \multirow[t]{3}{*}{3.0} & 441.20 & 426.93 & 404.13 & 2 & 50.890 & 41.814 & 36.055 & 4 \\
\hline & 392.35 & 397.81 & 397.18 & 0.01 & 34.013 & 34.013 & 34.491 & 0.7 \\
\hline & 364.70 & 394.62 & 397.14 & & 29.916 & 34.512 & 34.744 & \\
\hline \multirow[t]{3}{*}{3.5} & 311.80 & 311.36 & 311.34 & 0.02 & 15.673 & 13.998 & 12.988 & 2 \\
\hline & 311.67 & 311.51 & 311.45 & 0.01 & 12.650 & 12.809 & 12.785 & 0.06 \\
\hline & 311.51 & 311.44 & 311.41 & & 11.734 & 12.720 & 12.793 & \\
\hline \multirow[t]{3}{*}{4.0} & 311.33 & 311.33 & 311.33 & 0.03 & 7.8681 & 7.4009 & 7.1132 & 0.6 \\
\hline & 311.64 & 311.51 & 311.45 & 0.01 & 7.0494 & 7.0809 & 7.0698 & 0.01 \\
\hline & 311.50 & 311.44 & 311.41 & & 6.7753 & 7.0526 & 7.0707 & \\
\hline \multirow[t]{3}{*}{4.5} & 311.33 & 311.33 & 311.33 & 0.03 & 5.0494 & 4.9022 & 4.8125 & 0.1 \\
\hline & 311.64 & 311.51 & 311.45 & 0.01 & 4.80901 & 4.81646 & 4.81169 & 0.1 \\
\hline & 311.50 & 311.44 & 311.41 & & 4.7100 & 4.8007 & 4.8066 & \\
\hline \multirow[t]{3}{*}{5.0} & 311.33 & 311.33 & 311.33 & 0.03 & 3.5966 & 3.5509 & 3.5223 & 0.1 \\
\hline & 311.64 & 311.51 & 311.45 & 0.01 & 3.5334 & 3.5348 & 3.5317 & 0.1 \\
\hline & 311.50 & 311.44 & 311.41 & & 3.4897 & 3.5232 & 3.5268 & \\
\hline \multirow[t]{3}{*}{5.5} & 311.33 & 311.33 & 311.33 & 0.03 & 2.5679 & 2.5542 & 2.5454 & 0.2 \\
\hline & 311.64 & 311.51 & 311.45 & 0.01 & 2.5585 & 2.5576 & 2.5555 & 0.2 \\
\hline & 311.50 & 311.44 & 311.41 & & 2.5350 & 2.5497 & 2.5515 & \\
\hline \multirow[t]{3}{*}{6.0} & 311.33 & 311.33 & 311.33 & 0.03 & 1.6732 & 1.6695 & 1.6671 & 0.3 \\
\hline & 311.64 & 311.51 & 311.45 & 0.01 & 1.6771 & 1.6759 & 1.6746 & 0.2 \\
\hline & 311.50 & 311.44 & 311.41 & & 1.6637 & 1.6708 & 1.6718 & \\
\hline
\end{tabular}



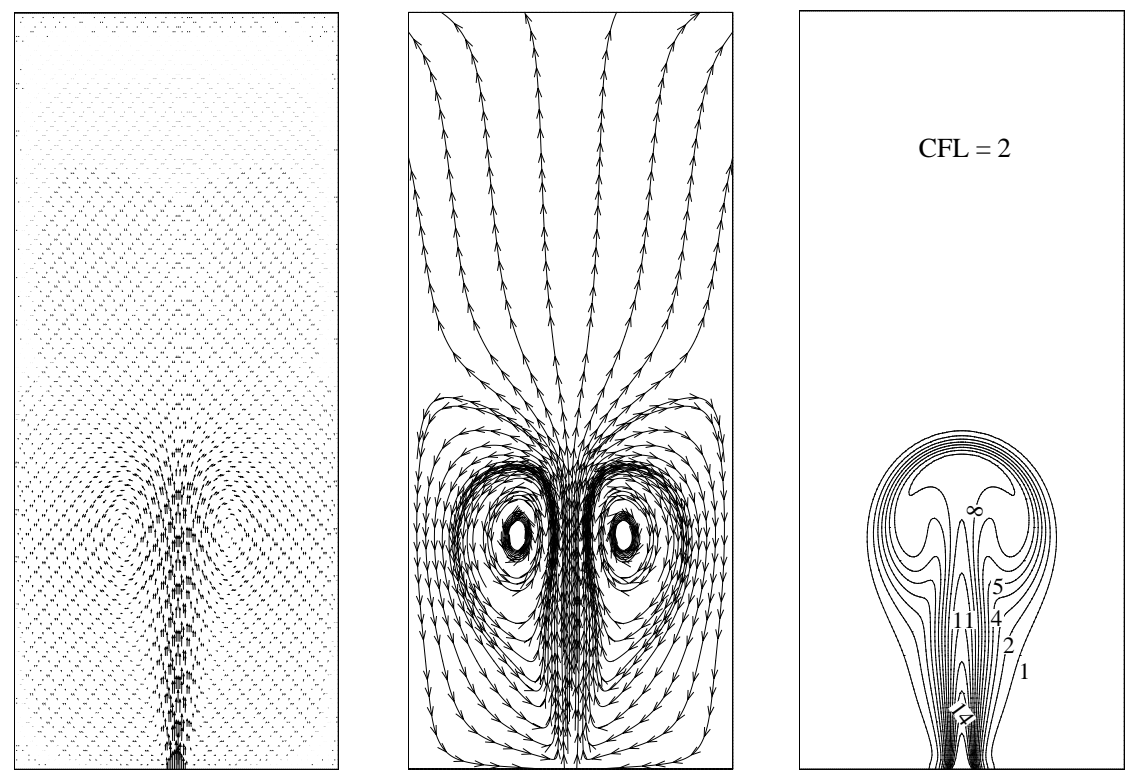

582
564
546
528
510
492
474
456
438
420
402
383
365
347
329

Figure 2. Case C1. From the left to the right: the velocity, the streamtraces and the temperature at $t=6 \mathrm{~s}$ (SI units).

divergence into two parts. The first term in the RHS of (7), is constant in space. It is linked to the variation of volume of each fluid element because of the isentropic compression caused by the incoming hot gas. At $t=6 \mathrm{~s}$, its value is

$$
-\frac{1}{\gamma P} \frac{\mathrm{d} P}{\mathrm{~d} t} \approx-\frac{1}{1.4 \cdot 113800} \frac{13800}{6} \mathrm{~s}^{-1} \approx-0.0144 \mathrm{~s}^{-1} ;
$$

This value is the value of the velocity divergence in the region in which there are no thermal effects due to the hot gas. As one can see in figure 3, there is a strong variation of the velocity divergence at the extremities of the injection region, where it reaches its maximum and minimum values (respectively about 2 and $-2 \mathrm{~s}^{-1}$ ). In this region the constant temperature hot gas enters into the cavity and the cold gas, drawn by the hot one, moves toward the hot one, as one can see in the streamtraces of figure 2 . Then the heat transport by convection (with the cold gas moving toward the hot one) counteracts to the heat diffusion and increases the strong variation of temperature (the temperature isolines are more dense at the extremities of the inlet than elsewhere). Moreover, since we impose a constant inlet temperature, we do not allow its variation by thermal diffusion, thus enhancing the temperature gradient at the extremities of the inlet. Even in the region far from the injection but reached by the injected gas, the velocity divergence cannot be considered constant: for instance in $x \approx 0$ and $y \approx 1$ the velocity divergence is about $-0.16 \mathrm{~s}^{-1}$, i.e. far from the value in the "undisturbed" region. We also note a large variation of the velocity divergence around the point $x=0$ and $y=3 \mathrm{~m}$, which corresponds to the region reached by the injected gas at $t=6 \mathrm{~s}$. It follows that the second term of the RHS of the divergence condition (7) cannot be neglected. 

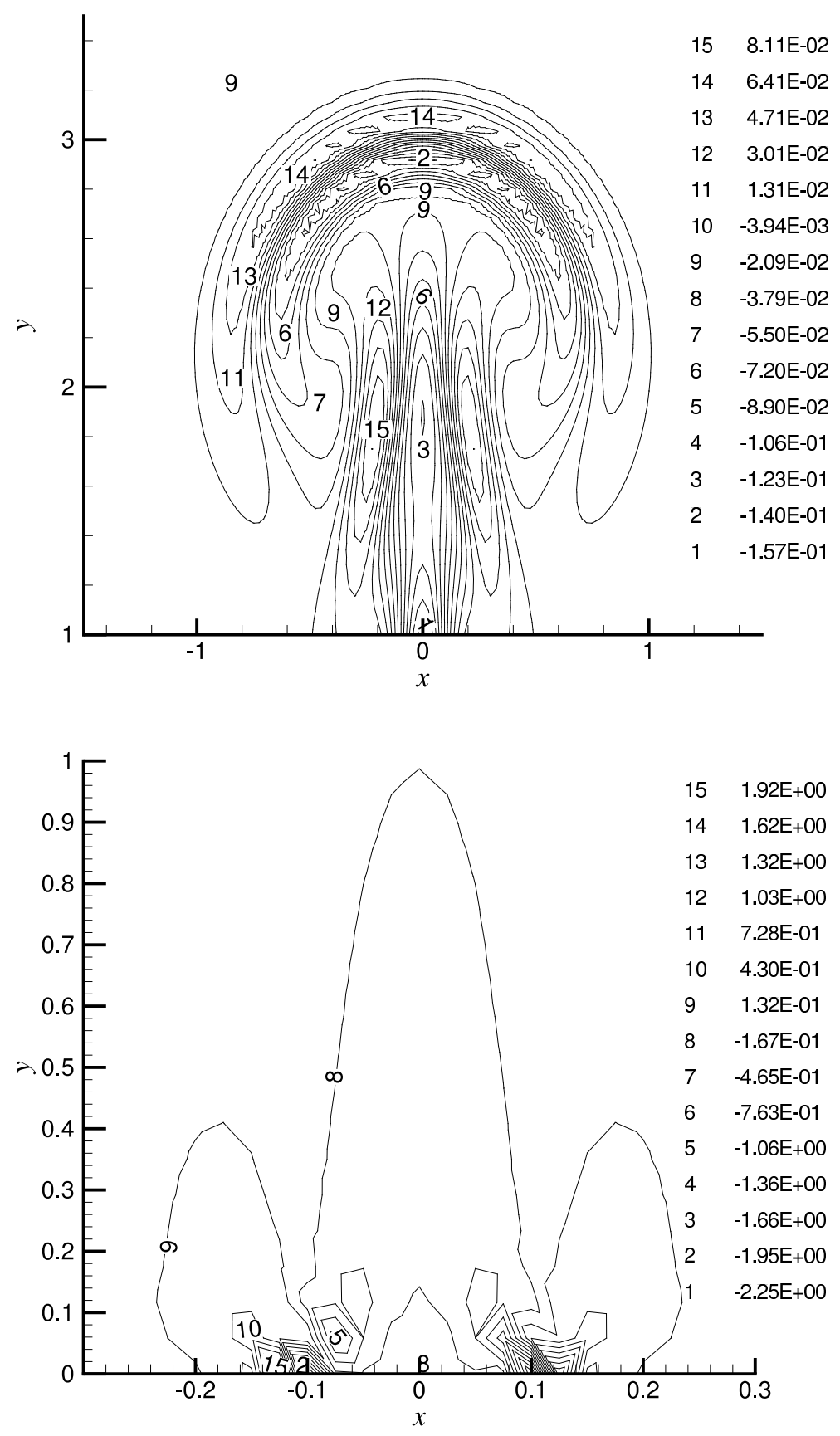

Figure 3. Case C1. The velocity divergence at $t=6 \mathrm{~s}$ (SI units). On the top, zoom close to the $y$-axis, in the region $1<y<3.5 \mathrm{~m}$. On the bottom, zoom close to the injection, in the region $y<1 \mathrm{~m}$. 

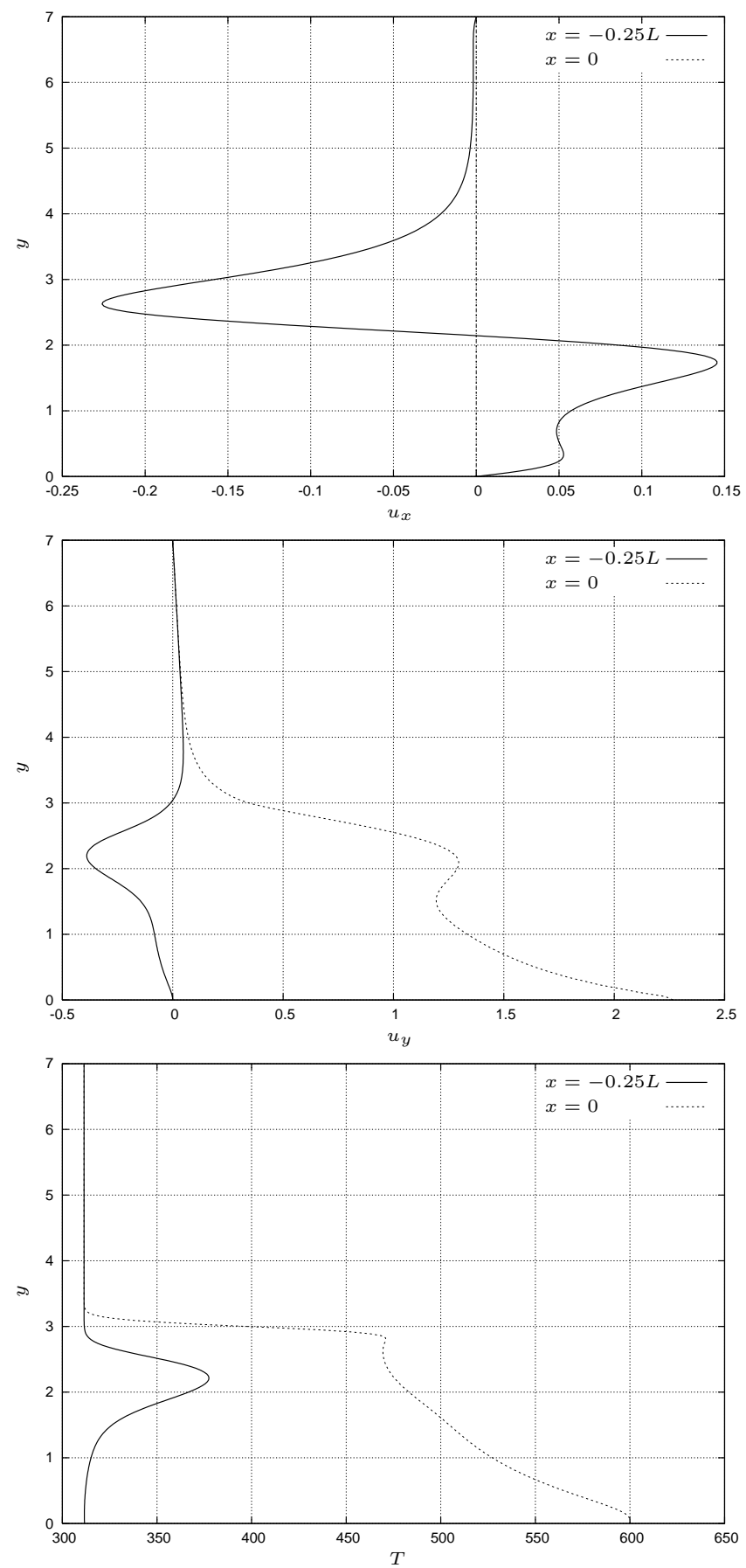

Figure 4. Case C1. Evolution of $u_{x}$ (top), $u_{y}$ (medium), $T$ (bottom) along the lines $x=0$ and $x=-L / 4$ at $t=6 \mathrm{~s}$. All the quantities are expressed in SI units. 

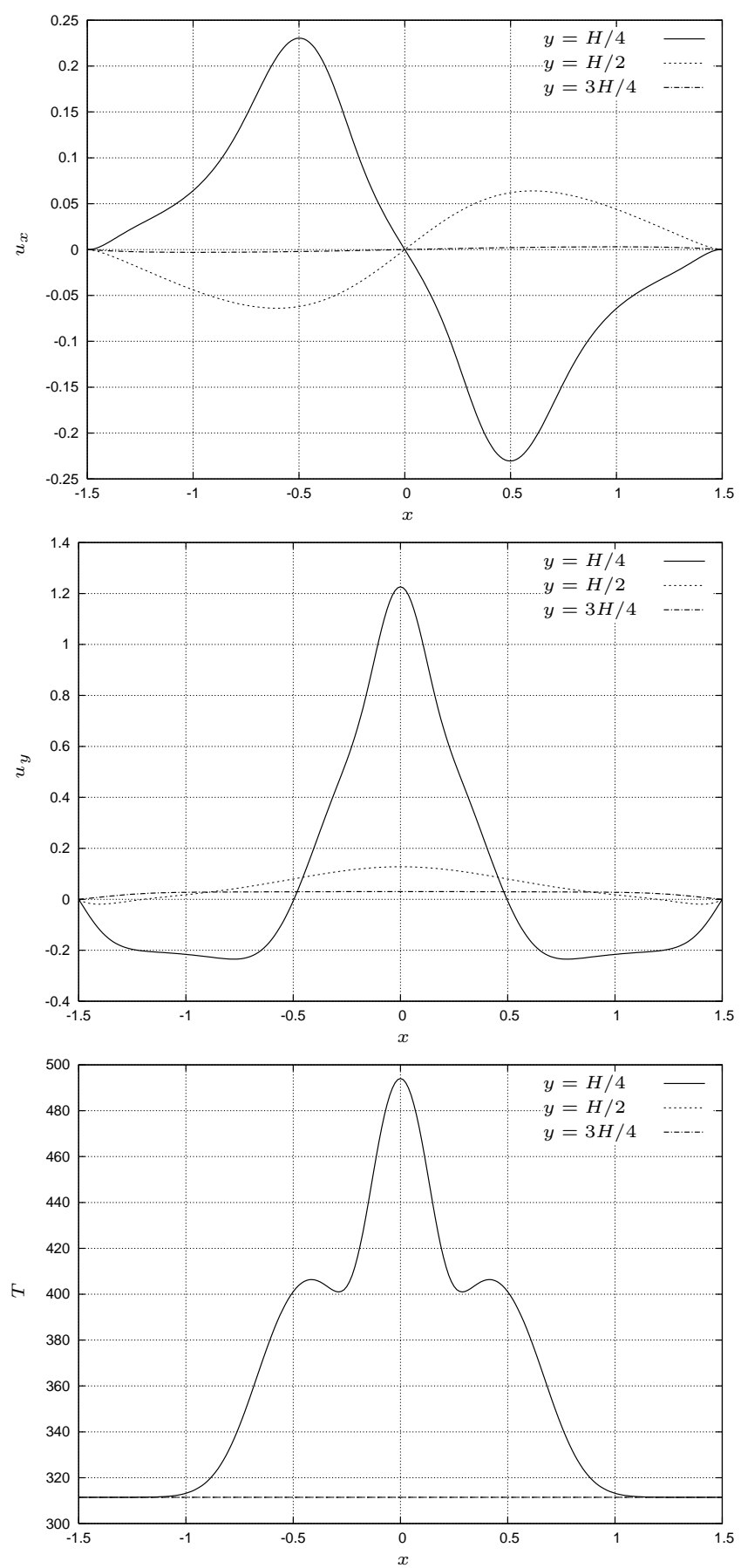

Figure 5. Case C1. Evolution of $u_{x}$ (top), $u_{y}$ (medium), $T$ (bottom) along the lines $y=H / 4, y=H / 2$ and $y=3 H / 4$ at $t=6 \mathrm{~s}$. All the quantities are expressed in SI units. 
The temperature isolines show that the temperature varies from $600 \mathrm{~K}$ at the injection to a constant value far from the injection. This constant value is $300 \mathrm{~K}$ at $t=0$ but increases because of the isentropic compression and it is about $311 \mathrm{~K}$ (formula (3) gives the same result) at $t=6 \mathrm{~s}$. In figure 4 we show the evolution of the temperature along the axis $x=0$. As one can see in this figure, as well as in the temperature isolines of figure 2 , close to the axis $x=0$ the temperature continuously decreases from 600 to $470 \mathrm{~K}$ from the inlet $(y=0 \mathrm{~m})$ to $y=3 \mathrm{~m}$. In this region, the decrease of temperature is mainly due to the mixing of the injected hot gas with the cold one arriving from the sides and transported by the vortices. Then, around $y=3 \mathrm{~m}$, we have an important variation of temperature, mainly due to the thermal diffusion.

As far as the velocity is concerned, the maximum value of $u_{y}$ as well as the maximum value of the speed occurs at the injection $(2.27 \mathrm{~m} / \mathrm{s})$. The maximum value of $u_{x}$ is about $0.4 \mathrm{~m} / \mathrm{s}$ and occurs at $( \pm 0.4,2.7) \mathrm{m}$, i.e. in the region close to the vortex.

6.1.2. Comparison of solvers. Let us now compare the results obtained using the different approaches. As far as the fully compressible solver is concerned, we compute the numerical solutions on three differently refined meshes. The coarsest one has $80 \times 80$ elements, the medium one $120 \times 120$, the finest $160 \times 160$. We take the $\mathrm{CFL}=2$ (the CFL being defined by formula (26), i.e. based on the flow velocity) and we use the BDF2 scheme for the time discretization. At each time step, fixed-point (internal) iterations are performed until the convergence error on the total energy decreases 5 orders of magnitude. Concerning the value of dual-time step $\left(\Delta \tau_{i}^{n, \ell}\right.$ in formula (14)), we take this value extremely high in order to remove the pseudo-time term. In our experience, this term is extremely useful in computing flows in open domains, in which the thermodynamic pressure does not vary in time. Indeed it enhance the condition number of the matrix involved in the quasi-Newton method, thus reducing the number of iterations performed by the GMRES solver. Conversely, in closed domains, this term prevents the time variation of thermodynamic pressure.

In the case of the asymptotic approaches, we compute the numerical solutions on a different set of refined meshes. The coarsest one has $40 \times 40$ elements, the medium one $80 \times 80$, the finest $120 \times 120$ (fewer elements than in the fully compressible approach, but more degrees of freedom). As far as the time-step is concerned, in order to obtain numerical results converged in time, we take a $\mathrm{CFL}=2$ (the CFL being defined by formula (26)) and we use the BDF2 scheme for the time discretization. At each time step, fixed-point (internal) iterations are performed until the convergence error on $T$ decreases 6 orders of magnitude. Streamline numerical diffusivity is not added (i.e. the convective term is treated with a pure Galerkin approach). The thermodynamic pressure is computed by imposing the conservation of the mass (equation (10)).

In table II we give the computed thermodynamic pressure. In the fully-compressible approach, the energy-balance is affected by the discretization error on the thermal diffusion; then the total pressure computed on the coarse mesh is slightly lower than the one computed on the medium and fine meshes. In the asymptotic approaches, the thermodynamic pressure is computed using the mass conservation instead of using the energy conservation. Nevertheless, the finer the mesh, the lower the difference of the thermodynamic pressure values computed using the different approaches. In table I we give some values of $T$ and $u_{y}$ on the axis $x=0$ at $t=6 \mathrm{~s}$, computed via the fully-compressible and the asymptotic algorithms. As one can see, the finer the mesh, the lower the differences between the values obtained using the different approaches. 
Table II. Case C1. Some values of the thermodynamic pressure at $t=6 \mathrm{~s}$ respectively given by the fully-compressible, the asymptotic 1 and the asymptotic 2 algorithms: grid convergence study.

\begin{tabular}{cccc}
\hline & \multicolumn{2}{c}{$P$ (bar) } & \\
Coarse & Medium & Fine & Eq $(2)$ \\
\hline 1.1384 & 1.1385 & 1.1385 & 1.1380 \\
1.1421 & 1.1405 & 1.1399 & $"$ \\
1.1407 & 1.1399 & 1.1395 & $"$ \\
\hline
\end{tabular}

We also show, on the fine meshes, the relative difference between the values obtained using the fully-compressible and the asymptotic approach 1 with respect to the asymptotic approach 2 , namely

$$
\begin{aligned}
\epsilon_{T} & =\frac{\left|T-T_{\text {ref }}\right|}{T_{\text {ref }}}, \\
\epsilon_{\mathrm{u}_{y}} & =\frac{\left|u_{y}-u_{y, \text { ref }}\right|}{\left|u_{y, \text { ref }}\right|},
\end{aligned}
$$

the reference values being the one given by the asymptotic approach 2 (which according to [10], should give the most accurate results). As one can see, for the fully-compressible approach $\epsilon_{T}$ goes from $0.03 \%$ to $2 \%$ and the maximum difference occurs at the strong gradient region at $y \approx 3 \mathrm{~m}$. For the asymptotic approach $1, \epsilon_{T}$ goes from $0.01 \%$ to $0.08 \%$. For the fullycompressible approach $\epsilon_{\mathrm{u}_{\mathrm{y}}}$ goes from $0.1 \%$ to $4 \%$ and the maximum difference occurs at the strong gradient region at $y \approx 3 \mathrm{~m}$. For the asymptotic approach $1, \epsilon_{T}$ goes from $0.05 \%$ to $0.1 \%$.

\subsection{Case C2: $g=9.81 \mathrm{~m} \mathrm{~s}^{-2}$ and $R e=40$.}

6.2.1. Solution analysis. We proceed as in the previous case, i.e. we present the numerical results at $t=6 \mathrm{~s}$ obtained via the asymptotic approach 1 using a regular mesh of $120 \times 120$ elements and a reference CFL number (always defined using formula (26)) equal to 2. Once again, the results obtained with the other approaches are very close to these.

In figure 6 we represent the velocity field, some streamtraces and the temperature field. In figure 7 we represent the divergence of $\vec{u}$. In figures 8 and 9 we show the evolution of $u_{x}, u_{y}$ and $T$ along some horizontal and vertical lines.

The computed thermodynamic pressure is 1.142 bar, very close to the one given using formula (2) (1.138 bar), which does not take into account the heat flux at the injection due to thermal convection.

As in the previous case (case $\mathrm{C} 1$ ), the solution is symmetric with respect to the $y$-axis. But in this latter case, we note that the hot region has reached the top of the cavity. This is due to the buoyancy force, which is very important with respect to the inertial force linked to the injection, according to the fact that the Froude number is equal to 0.042 . In the case $\mathrm{C} 1$, at $t=6 \mathrm{~s}$, the maximum values of $u_{y}$ at the injection occurs at the $y$-axis, and it is equal to $2.2 \mathrm{~m} / \mathrm{s}$. In the case $\mathrm{C} 2$, at $t=6 \mathrm{~s} u_{y}$ at the injection is equal to $2.2 \mathrm{~m} / \mathrm{s}$ (as in the case $\mathrm{C} 1$, since this is due to the injection boundary conditions). Nevertheless, the maximum value of $u_{y}$ 
Table III. Case C2. Some values (SI units) along the axis $x=0$ at $t=6 \mathrm{~s}$ respectively given by the fully-compressible, the asymptotic 1 and the asymptotic 2 algorithms: grid convergence study. $\epsilon_{T}$ and $\epsilon_{\mathrm{u}_{\mathrm{y}}}$ are defined by equation (27).

\begin{tabular}{|c|c|c|c|c|c|c|c|c|}
\hline$y$ & Coarse & $\begin{array}{c}T \\
\text { Medium }\end{array}$ & Fine & $\begin{array}{c}\epsilon_{T}(\%) \\
\text { Fine }\end{array}$ & Coarse & $\begin{array}{l}u_{y} \cdot 100 \\
\text { Medium }\end{array}$ & Fine & $\begin{array}{c}\epsilon_{\mathrm{u}_{\mathrm{y}}}(\%) \\
\text { Fine }\end{array}$ \\
\hline \multirow[t]{3}{*}{0.5} & 514.36 & 517.80 & 519.78 & 1 & 2.6771 & 2.7615 & 2.7990 & 2 \\
\hline & 527.17 & 525.82 & 525.64 & 0.02 & 2.8586 & 2.8608 & 2.8611 & 0.02 \\
\hline & 529.16 & 526.17 & 525.73 & & 2.8885 & 2.8617 & 2.8617 & \\
\hline \multirow[t]{3}{*}{1.0} & 458.41 & 460.65 & 461.87 & 0.8 & 3.0327 & 3.0954 & 3.1241 & 2 \\
\hline & 465.71 & 465.88 & 465.71 & 0.002 & 3.1998 & 3.1813 & 3.1812 & 0.01 \\
\hline & 466.55 & 465.92 & 465.70 & & 3.1956 & 3.1816 & 3.1816 & \\
\hline \multirow[t]{3}{*}{1.5} & 429.53 & 431.20 & 432.07 & 0.6 & 3.2661 & 3.3174 & 3.3415 & 2 \\
\hline & 434.81 & 435.10 & 434.95 & 0.005 & 3.3871 & 3.3957 & 3.3955 & 0.06 \\
\hline & 435.17 & 435.03 & 434.93 & & 3.4088 & 3.3968 & 3.3957 & \\
\hline \multirow[t]{3}{*}{2.0} & 411.84 & 413.15 & 413.84 & 0.6 & 3.4510 & 3.4977 & 3.5197 & 2 \\
\hline & 415.91 & 416.29 & 416.16 & 0.007 & 3.5566 & 3.5765 & 3.5759 & 0 \\
\hline & 416.18 & 416.22 & 416.13 & & 3.5907 & 3.5784 & 3.5759 & \\
\hline \multirow[t]{3}{*}{2.5} & 399.86 & 400.91 & 401.47 & 0.5 & 3.5975 & 3.6458 & 3.6689 & 2 \\
\hline & 403.07 & 403.54 & 403.43 & 0.007 & 3.7177 & 3.7329 & 3.7316 & 0.008 \\
\hline & 403.37 & 403.48 & 403.40 & & 3.7411 & 3.7323 & 3.7313 & \\
\hline \multirow[t]{3}{*}{3.0} & 391.45 & 392.33 & 392.78 & 0.4 & 3.6649 & 3.7081 & 3.7311 & 1 \\
\hline & 393.77 & 394.57 & 394.50 & 0.01 & 3.8128 & 3.7854 & 3.7803 & 0.01 \\
\hline & 394.31 & 394.57 & 394.46 & & 3.7866 & 3.7699 & 3.7799 & \\
\hline \multirow[t]{3}{*}{3.5} & 385.54 & 386.40 & 386.80 & 0.5 & 3.6532 & 3.6494 & 3.6510 & 0.2 \\
\hline & 386.98 & 388.50 & 388.49 & 0.09 & 3.8244 & 3.6582 & 3.6435 & 0.03 \\
\hline & 387.94 & 388.57 & 388.45 & & 3.7102 & 3.6420 & 3.6447 & \\
\hline \multirow[t]{3}{*}{4.0} & 380.35 & 381.44 & 381.98 & 0.5 & 3.9013 & 3.9083 & 3.9119 & 2 \\
\hline & 382.14 & 383.74 & 383.77 & 0.01 & 3.8465 & 3.9812 & 3.9980 & 0.07 \\
\hline & 382.92 & 383.67 & 383.73 & & 4.0740 & 4.0434 & 3.9983 & \\
\hline \multirow[t]{3}{*}{4.5} & 373.31 & 374.48 & 375.14 & 0.4 & 4.6538 & 4.7651 & 4.8090 & 3 \\
\hline & 377.65 & 376.88 & 376.69 & 0.008 & 4.4967 & 4.9489 & 4.9789 & 0.05 \\
\hline & 377.03 & 376.47 & 376.66 & & 4.9753 & 5.0130 & 4.9763 & \\
\hline \multirow[t]{3}{*}{5.0} & 366.06 & 366.97 & 367.47 & 0.3 & 5.2890 & 5.3985 & 5.4430 & 2 \\
\hline & 371.71 & 368.93 & 368.61 & 0.005 & 5.3291 & 5.5542 & 5.5598 & 0.005 \\
\hline & 369.43 & 368.50 & 368.59 & & 5.5340 & 5.5643 & 5.5601 & \\
\hline \multirow[t]{3}{*}{5.5} & 361.04 & 361.51 & 361.80 & 0.2 & 3.4510 & 3.4977 & 5.5045 & 1 \\
\hline & 365.15 & 362.97 & 362.64 & 0.008 & 5.6427 & 5.5745 & 5.5668 & 0.07 \\
\hline & 363.23 & 362.65 & 362.61 & & 5.4869 & 5.5347 & 5.5707 & \\
\hline \multirow[t]{3}{*}{6.0} & 357.86 & 358.05 & 358.18 & 0.2 & 4.9469 & 5.0068 & 5.0280 & 0.005 \\
\hline & 360.31 & 359.22 & 358.91 & 0.008 & 5.2202 & 5.0257 & 5.0236 & 0.08 \\
\hline & 359.27 & 359.02 & 358.88 & & 4.8862 & 4.9760 & 5.0277 & \\
\hline
\end{tabular}



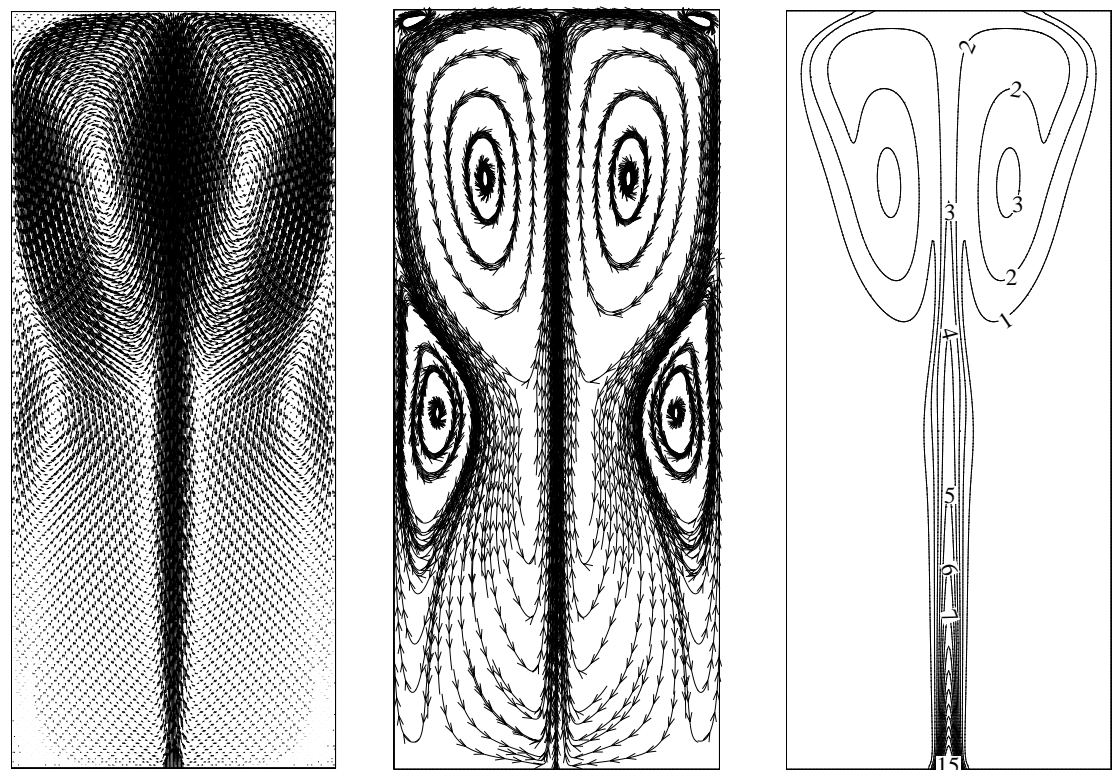

$$
\begin{array}{ll}
15 & 582 \\
14 & 564 \\
13 & 546 \\
12 & 528 \\
11 & 510 \\
10 & 492 \\
9 & 474 \\
8 & 456 \\
7 & 438 \\
6 & 420 \\
5 & 402 \\
4 & 383 \\
3 & 365 \\
2 & 347 \\
1 & 329
\end{array}
$$

Figure 6. Case $\mathrm{C} 2$. From the left to the right: the velocity, the streamtraces and the temperature at $t=6 \mathrm{~s}$ (SI units).

on the $y$-axis is $5.6 \mathrm{~m} / \mathrm{s}$ and occurs at $y \approx 5.3 \mathrm{~m}$. The maximum value of $u_{x}$ is about $3.5 \mathrm{~m} / \mathrm{s}$ and occurs at $(x, y)=( \pm 0.6,6.9) \mathrm{m}$, i.e. on the top of the cavity. Here, the hot gas arrives and deviates on the left and on the right, thus generating a significant velocity in horizontal direction.

As far as the velocity divergence is concerned, as in the case $\mathrm{C} 1$ it cannot be considered constant in space. Indeed the constant part of the velocity divergence is given by

$$
-\frac{1}{\gamma P} \frac{\mathrm{d} P}{\mathrm{~d} t} \approx-\frac{1}{1.4 \cdot 114100} \frac{14100}{6} \mathrm{~s}^{-1} \approx 0.0147 \mathrm{~s}^{-1} .
$$

Its extremal values are -3.7 and $4.2 \mathrm{~s}^{-1}$ and occurs at the extremities of the inlet.

The temperature isolines show that the temperature varies from $600 \mathrm{~K}$ at the injection to a constant value far from the injection $(311 \mathrm{~K})$, as in the case $\mathrm{C} 1$. The quantity of hot gas entering the cavity is the same as in the case $\mathrm{C} 1$ but, because of the buoyancy forces, its shape is different. In the former case, the hot gas is around the inlet, while in this case it moves faster toward the top in a narrow region close to the $y$-axis. In figure 8 we show the evolution of the temperature along the axis $x=0$ and in figure 9 we shows its evolution on some horizontal lines. As one can see in this figure, as well as in the temperature isolines of figure 6 , close to the axis $x=0$ the temperature continuously decreases from 600 to $360 \mathrm{~K}$ from the inlet to top. In this narrow region around the $y$-axis, the decrease of temperature is mainly due to the mixing of the injected hot gas with the cold one arriving from the sides and transported by the vortices. Note that, because of the buoyancy force, the velocity values are larger than in the case $\mathrm{C} 1$. The larger the velocity values, the larger the mixing, the more significant the 

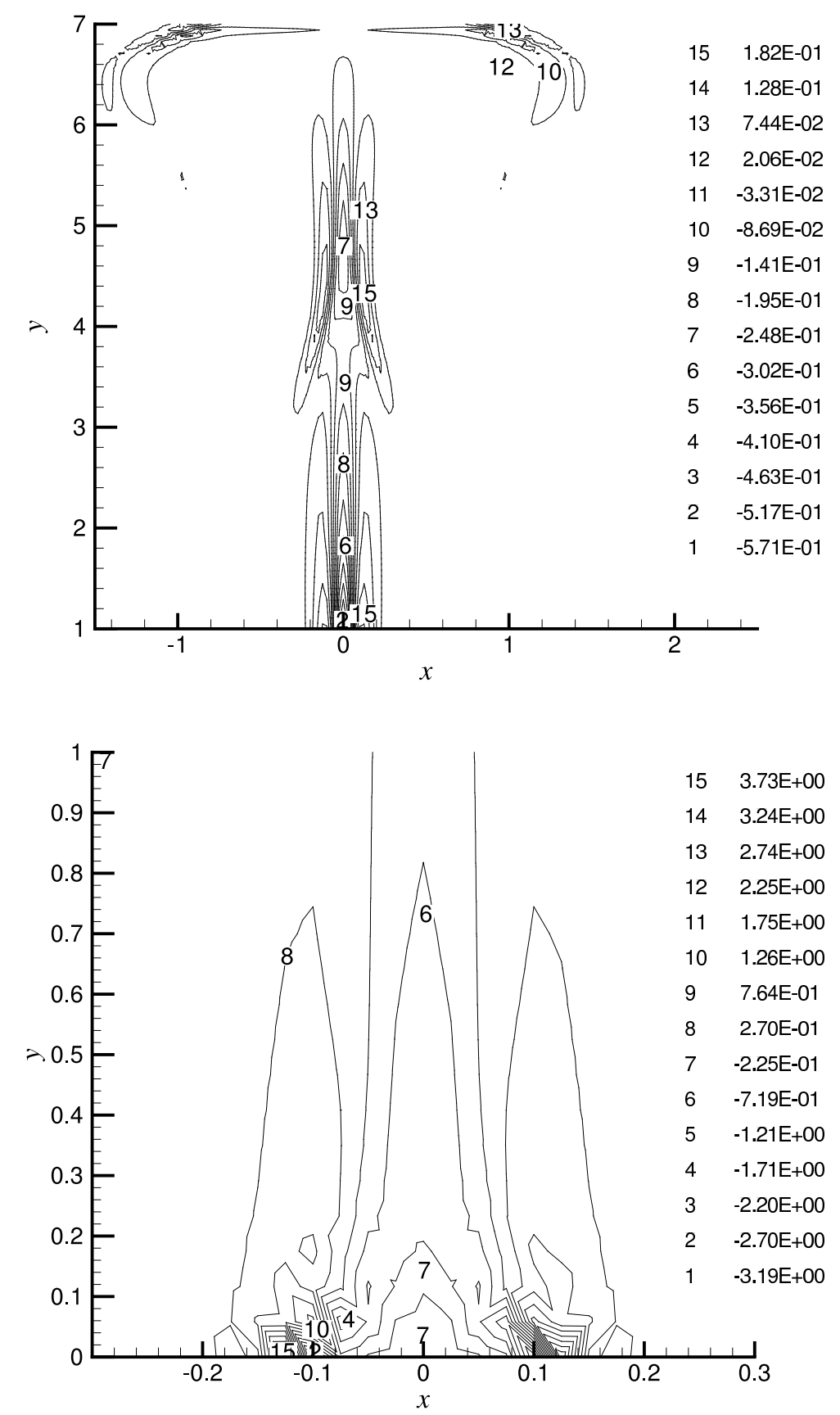

Figure 7. Case C2. The velocity divergence at $t=6 \mathrm{~s}$ (SI units). On the top, the whole region. On the bottom, zoom close to the injection, in the region $y<1 \mathrm{~m}$. 

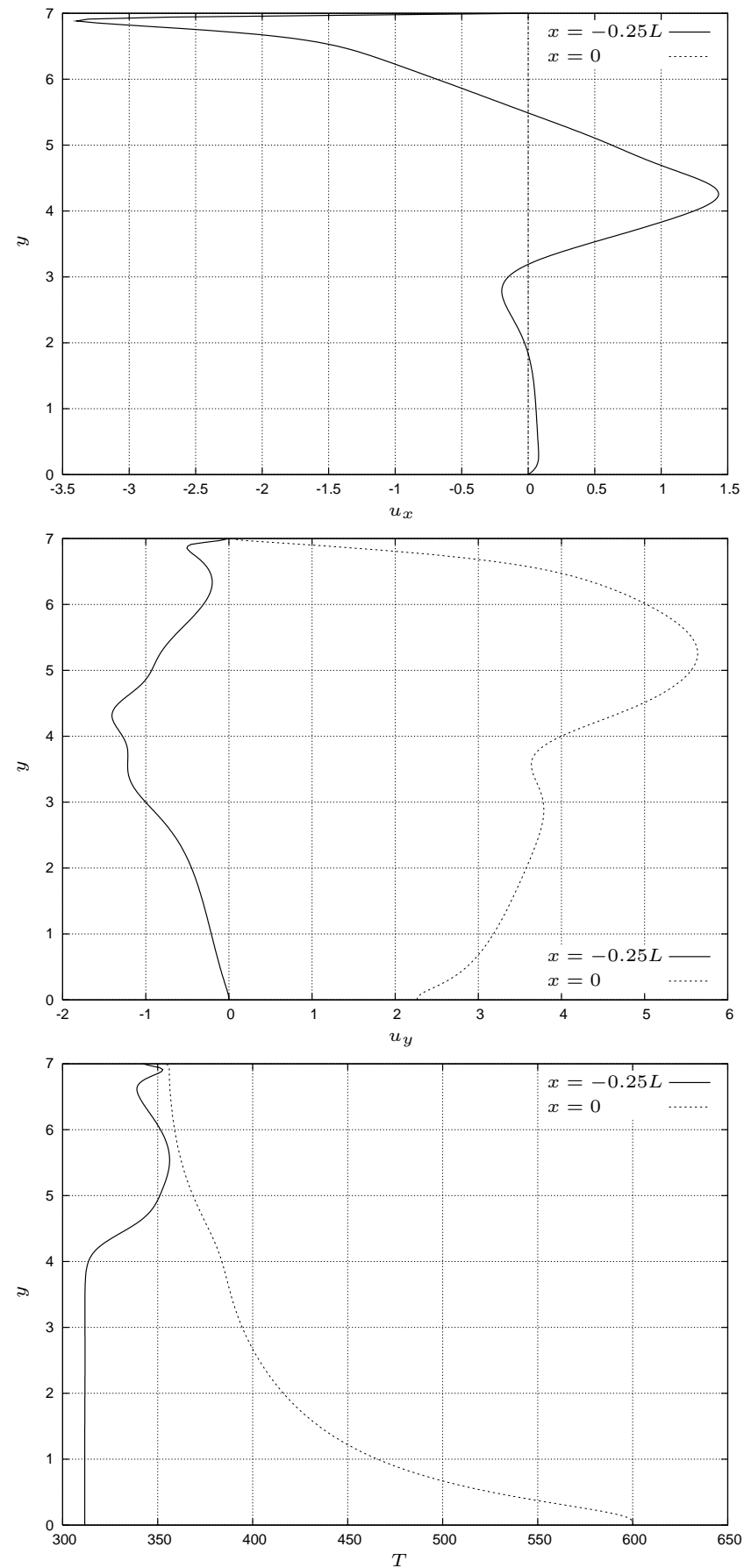

Figure 8. Case C2. Evolution of $u_{x}$ (top), $u_{y}$ (medium), $T$ (bottom) along the lines $x=0$ and $x=-L / 4$ at $t=6 \mathrm{~s}$. All the quantities are expressed in SI units. 

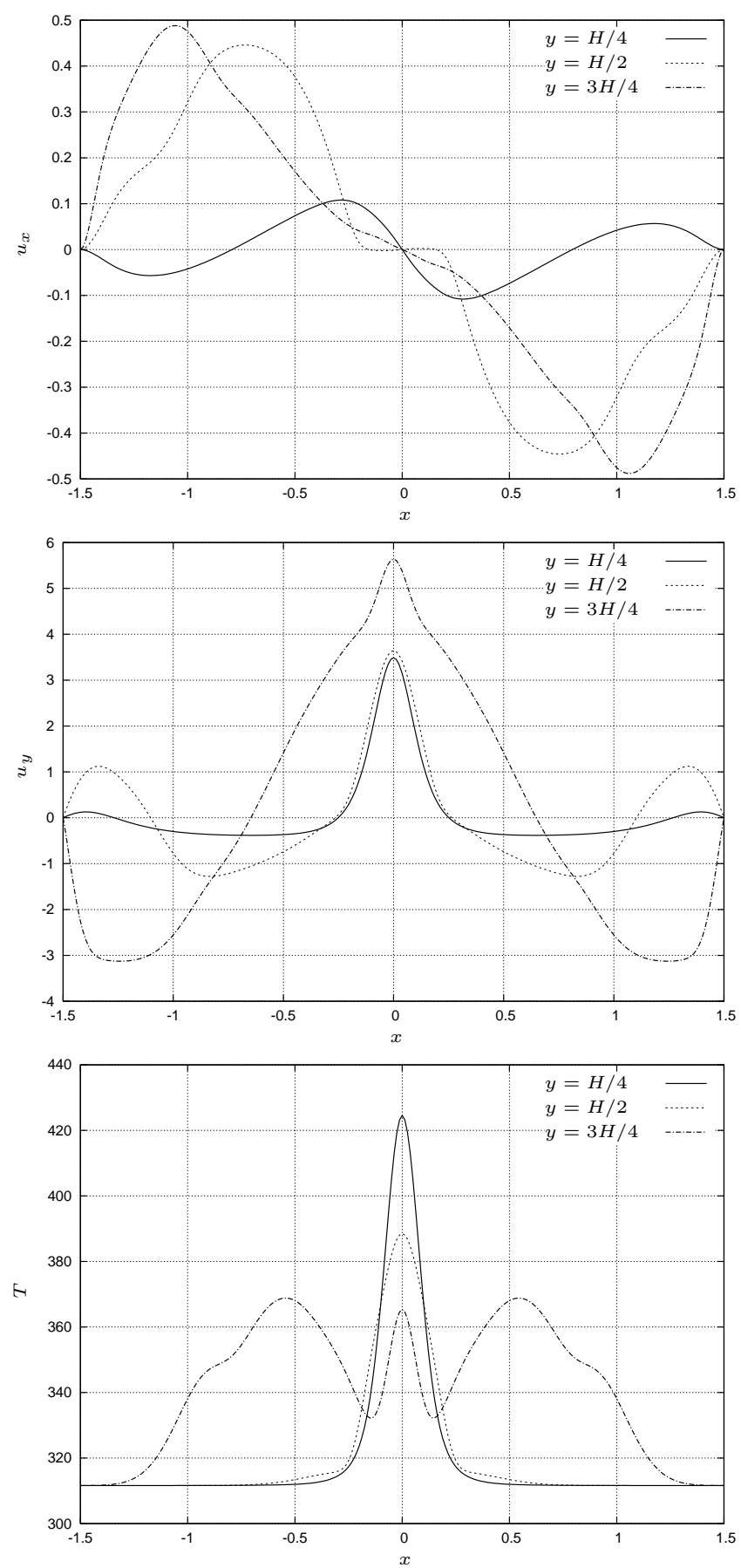

Figure 9. Case C2. Evolution of $u_{x}$ (top), $u_{y}$ (medium), $T$ (bottom) along the lines $y=H / 4, y=H / 2$ and $y=3 H / 4$ at $t=6 \mathrm{~s}$. All the quantities are expressed in SI units. 
Table IV. Case C2. Some values of the thermodynamic pressure at $t=6 \mathrm{~s}$, respectively given by the fully-compressible, the asymptotic 1 and the asymptotic 2 algorithms: grid convergence study.

\begin{tabular}{cccc}
\hline & \multicolumn{2}{c}{$P$ (bar) } & \\
Coarse & Medium & Fine & Eq $(2)$ \\
\hline 1.1385 & 1.1387 & 1.1389 & 1.1380 \\
1.1444 & 1.1429 & 1.1419 & $"$ \\
1.1447 & 1.1427 & 1.1417 & $"$ \\
\hline
\end{tabular}

Table V. Case C2. CPU time (s) spent on a Linux PC after 6 s of physical time.

\begin{tabular}{c|ccc}
\hline Approach & \multicolumn{3}{|c}{ CPU time (s) } \\
& Coarse & Medium & Fine \\
\hline Fully-comp. & 7850 & 38500 & 105000 \\
Asymptotic 2 & 2450 & 23400 & 105000 \\
\hline
\end{tabular}

temperature variation.

6.2.2. Comparison of solvers. Let us now compare the results obtained using the different approaches. In table IV we represent the computed thermodynamic pressure. Note the fullycompressible approach and the asymptotic ones converge to a value between 1.39 and 1.41 bar. Once again, the finer the mesh, the lower the difference between the values given by the different approaches. In table III we represent some values of $T$ and $u_{y}$ on the the axis $x=0$ at $t=6 \mathrm{~s}$, computed via the fully-compressible and the asymptotic algorithms. On the fine grids the relative differences between the fully-compressible approach and the asymptotic approach 2 are less than $1 \%$ for the temperature and less than $3 \%$ for the velocity; the relative differences between the asymptotic approach 1 and the asymptotic approach 2 are less than $0.1 \%$ for the temperature and for the velocity.

\section{PERFORMANCE AND SENSITIVITY ANALYSIS}

In this section we investigate the performances of the fully-compressible approach and of the asymptotic solver 2 (concerning the asymptotic solver 1 , we only report that its performances are slightly better than the ones of the asymptotic solver 2, because on a mesh with the same number of elements the number of degrees of freedom for the dynamic pressure are fewer; see [10] for the discussion on conformal P1 versus non-conformal P1 elements for the dynamic pressure). We also perform the sensitivity analysis of the solution with respect to the variation of some numerical parameters which allow to easily and drastically reduce the CPU time consumption.

In table $\mathrm{V}$, we show the CPU-time consumption of the two approaches on a LINUX PC with two Intel Xeon Processors with a CPU frequency of $3 \mathrm{GHz}$ and with $2 \mathrm{Mb}$ of RAM memory. 
We emphasize that, in practical applications, our calculations involve 3D domains and several hours of physical time. Then a problem arises: how to reduce the CPU time consumption.

Concerning the fully compressible approach, we recall that it is theoretically second order accurate in space. The number of degrees of freedom are linear with the number of elements. In the coarse, medium and fine mesh, the degrees of freedom are respectively $80 \times 80 \times 4$, $120 \times 120 \times 4$ and $160 \times 160 \times 4(25600,57600,102400)$. In the asymptotic approach 2 , we expect to be third order accurate for the velocity and the temperature and second order accurate for the dynamic pressure. The degrees of freedom for each component of the velocity and for the temperature are about $4 \times 40 \times 40,4 \times 80 \times 80,4 \times 120 \times 120(6400,25600,57600)$; the degree of freedom for the dynamic pressure are $3 \times 40 \times 40,3 \times 80 \times 80,3 \times 120 \times 120$ $(4800,19200,43200)$; i.e. the total number of degrees of freedom are about 24000,96000 , 216000. Numerical experiments confirm that in the fully compressible approach, the results obtained are less accurate than the ones obtained with the asymptotic approach and its CPU consumption is greater. This is due to the fact that, in the fully-compressible approach, the governing equations are solved simultaneously and all the conservative variables are coupled, while in the asymptotic approach we solve two separate systems (one for the velocity and the dynamic pressure, the other for the temperature). Moreover the matrix involved in the linear system of the quasi-Newton method is ill-conditioned, and this is due to the fact that the time step is much larger than the time that the acoustic waves take to cross a mesh. In the literature, this problem has been solved using the dual-time preconditioning matrix. As already mentioned, in problems in which the thermodynamic pressure is time-dependent this approach cannot be used since it prevents the thermodynamic pressure variation. The storage of the matrix involved in the linear system of the quasi-Newton method is also a problem. In [12], an efficient algorithm is presented which avoids the storage of this matrix and allows one to save the CPU-time with respect to the fully-compressible approach. This approach efficiently works in dealing with open domains (in which the thermodynamic pressure stays constant) but presents some problems in closed domains. Nevertheless, even in a problem on an open domain, like the study of a tee junction, the CPU-time consumption is greater than in the asymptotic approach.

CPU-time consumption of the asymptotic approach can be reduced by increasing the CFL number and/or reducing the non-linear iterations. For instance, if we take the CFL number (defined by (26)) equal 20 (ten times larger than the one previously used), the number of non-linear iterations (involving the index $\ell$ in the algorithm presented in section 5) as well as the number of linear iterations in the GMRES solver are almost the same as in the case of CFL equal 2; then the CPU time is reduced by a factor 10. If, instead of performing non-linear iterations to reduce the error on temperature of a factor $10^{5}$ (which require around 10 and 15 iteration over the index $\ell$ ), we just perform one non-linear iteration, this also drastically reduces the CPU time (of a factor larger than 10). Finally we can both increase the CFL and just perform one non-linear iteration. In figure 10 we show the results obtained using all these different approaches on the medium grid (which, as we have seen in table III, already gives accurate results). From the top to the bottom and from the left to the right, we have shown the temperature isolines for the following cases.

1. The solution obtained with the approach described in the previous section (namely with the BDF2 scheme for time discretization and iterating over the index $\ell$ ).

2. A first order accurate solution in time (implicit Euler scheme or BDF1 for time 
discretization).

3. A second order accurate solution in time with $\mathrm{CFL}=20$ (and iterating over the index $\ell$ ).

4. A solution obtained using the BDF2 scheme but without iterating over the index $\ell$ (with $\mathrm{CFL}=2$ ).

5. A solution obtained using the BDF2 scheme with $\mathrm{CFL}=20$ without iterating over the index $\ell$.

6. A solution obtained with the BDF2 scheme for time discretization, with $\mathrm{CFL}=2$, iterating over the index $\ell$ but with lumped mass matrices.

Cases 2 and 6 are considered for comparisons, because they do not involve any CPU time reduction; nevertheless the result on the case 6 becomes interesting if fractional step with explicit approach for the convection is used. The cases closest to the reference solution are respectively the case 6 and 2. Note that case 4 can also be interpreted as a fractional step approach: we first determine the velocity together with the dynamic pressure and then the temperature. This fractional steps approach gives results which are much less accurate that the ones obtained with case 2 (BDF1 for time discretization) and case $3(\mathrm{CFL}=20$ but non-linearity correctly taken into account): in case 4 the hot gas has not reached the top of the cavity. This shows the importance of the coupling between the temperature and the velocity (via the coefficients in the equation for the velocity but also via the temperature in the divergence condition). Case 5 gives results which are completely different from the converged ones (the mass of hot gas is still at the bottom of the cavity).

We emphasize that, in 3D applications, the storage of the matrix of the problem involving the velocity and the dynamic pressure can be a problem. It follows that fractional steps, in which thermodynamic pressure and velocity are solved separately, are necessary. Some models have been proposed in the literature (see for instance [18]). Nevertheless a validation on injection test cases is necessary, as confirms the loss of accuracy in case 4. 


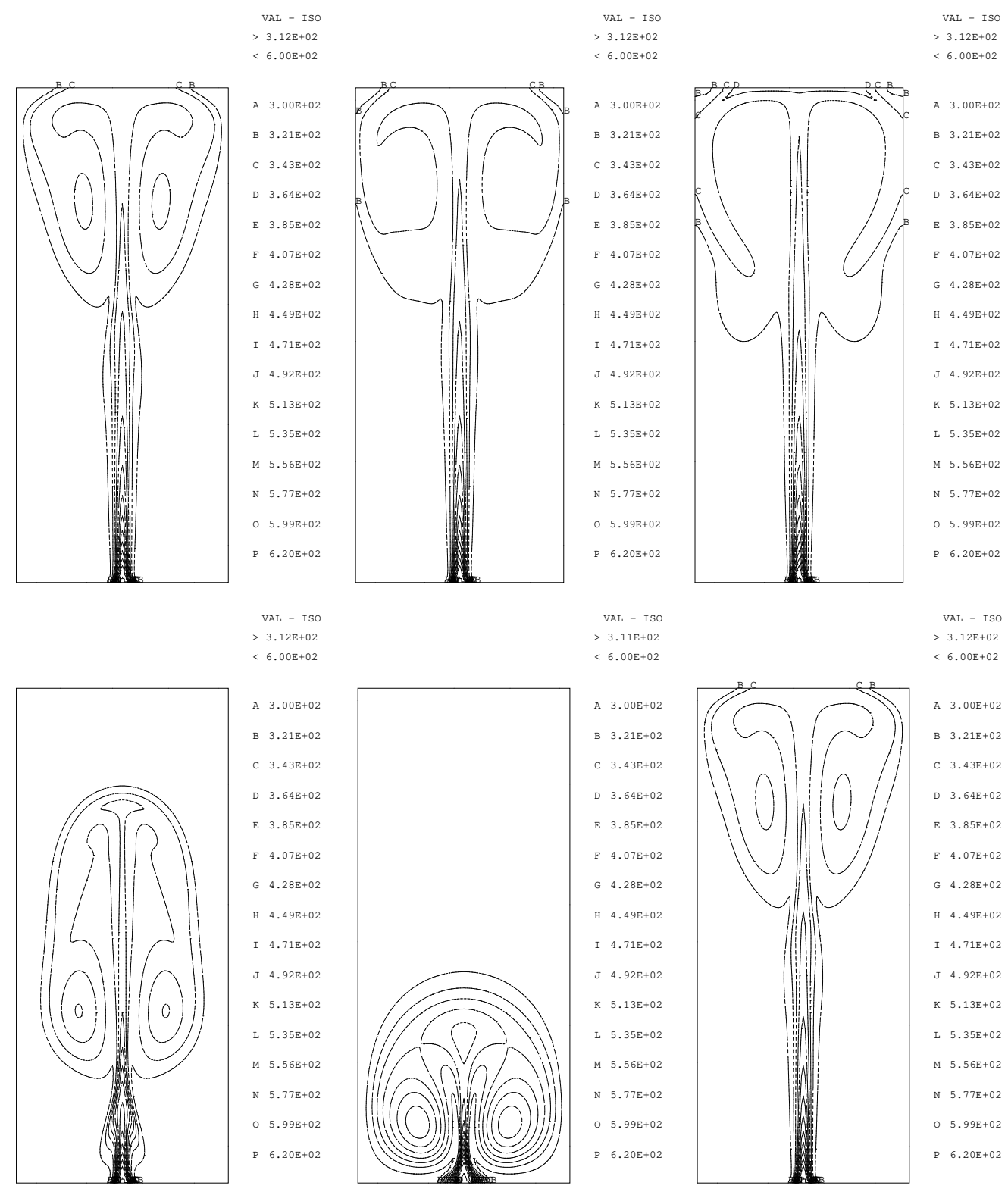

Figure 10. Case C2. Asymptotic approach 2. Sensitivity analysis. Temperature isolines at $t=6 \mathrm{~s}$. From the top to the bottom and from the left to the right: reference solution, solution first-order accurate in time (BDF1), solution for $\mathrm{CFL}=20$, solution for $\mathrm{CFL}=2$ without performing non-linear iterations, solution for $\mathrm{CFL}=20$ without performing non-linear iterations, solution with lumped mass matrices. 


\section{CONCLUSION}

In this work, we have investigated a problem of injection of hot gas into a cavity filled with the same gas. Three different approaches have been used: a fully-compressible approach (densitybased solver) and two asymptotic approaches (pressure-based solvers), which differ from one another on the number of variables involved and the way in which the divergence condition is taken into account. The convergence analysis shows that the different solvers give results very close to one another, thus proving the accuracy of the results here provided.

The fully compressible approach gives results which are less accurate than the ones obtained with the other approaches. Moreover it takes more CPU time. This is due to the fact that, in the former case, all the equations are solved simultaneously and the matrix arising from the quasi-Newton method is ill-conditioned. However, the fully compressible solver can solve flows at all speeds, while asymptotic approaches solve a system of equations arising from one-time scale and one-space scale asymptotic analysis. Future work will concern the improvement of the fully compressible solver by implementing a Jacobian-free implicit which avoids the storage of the matrix arising from the quasi-Newton method and of its preconditioning matrix, with the same strategy as the one presented in [14].

Concerning the asymptotic approaches it has been shown that, if the non-linear coupling between the temperature and the velocity, due to the coefficients appearing in the velocity equation but also in the divergence condition, is not correctly solved, the loss of accuracy is significant. Nevertheless, the fixed-point iteration procedure used to solve this non-linear coupling increases the CPU time-consumption (of a factor larger than 10). As already mentioned, in 3D applications, the storage of the matrix of the problem involving the velocity and the dynamic pressure can be a problem. It follows that fractional steps, in which thermodynamic pressure and velocity are solved separately, are necessary. Some models have been proposed in the literature (see for instance [18]). Nevertheless a validation on injection test cases is necessary, as confirms the investigation performed in the previous section, and will be the object of a future work.

\section{ACKNOWLEDGEMENTS}

This work has been supported by the CEA objective direction DSOE in the framework of the MATHY project.

The authors gratefully acknowledge the anonymous referees for their comments and their suggestions.

A special thank is addressed to Donna Calhoun, for several suggestions and corrections concerning the English language, and to Thierry Charras, Alain Millard and Pierre Verpeaux, for their work in maintaining the CAST3M code.

\section{APPENDIX. Asymptotic analysis and asymptotic models (6) and (11)}

In this section we explain in details how the asymptotic systems of equations (6) and (11) are obtained from the compressible Navier-Stokes equations via the single time scale and single space scale asymptotic analysis. The single time scale and single space scale asymptotic analysis of the compressible Navier-Stokes equations at low Mach number regime can be found 
in many other works (see for instance [22], in which Paolucci derives the low Mach number Navier-Stokes equations used in [5] to compute the natural convection in a square cavity with large temperature differences). Nevertheless, the purpose of this appendix is to clarify which terms are neglected in the asymptotic models here considered. We emphasize that we follow here the same approach as in [35]; i.e., using the same reference scales as in [35], we write in non-dimensional form the equation of conservation of internal energy and the time-evolution equations for the velocity and for the temperature. Once again, we restrict our attention to a calorically perfect gas, with constant thermal diffusivity $\lambda$ and dynamic viscosity $\mu$.

\subsection{Non-dimensional compressible Navier-Stokes equations}

The compressible Navier-Stokes equations can be written in the conservative form (4). As noticed in [35], chapter 1, page 16, (see also [3], chapter VI, for details), combining these equations, we can get

- the equation of conservation of internal energy

$$
\frac{\partial \rho e}{\partial t}+\vec{\nabla} \cdot(\rho \vec{u} e)+p \vec{\nabla} \cdot \vec{u}-\underline{\tau}: \vec{\nabla} \vec{u}-\vec{\nabla} \cdot(\lambda \vec{\nabla} T)=0
$$

where

$$
e=e_{t}-\frac{1}{2} \vec{u} \cdot \vec{u}=\frac{1}{\gamma-1} \frac{P}{\rho}
$$

- the time-evolution equation for the temperature

$$
\rho c_{p}\left(\frac{\partial T}{\partial t}+\vec{u} \cdot \vec{\nabla} T\right)-\left(\frac{\partial p}{\partial t}+\vec{u} \cdot \vec{\nabla} p\right)-\underline{\tau}: \vec{\nabla} \vec{u}-\vec{\nabla} \cdot(\lambda \vec{\nabla} T)=0
$$

- the time-evolution equation for the velocity

$$
\rho \frac{\partial \vec{u}}{\partial t}+\rho \vec{u} \cdot \vec{\nabla} \vec{u}=-\vec{\nabla} p+\vec{\nabla} \cdot \underline{\tau}+\rho \vec{g} .
$$

Let us now write equations (4), (28), (29) and (30) in non-dimensional form. For density, velocity, length and pressure we take as reference scales $\rho_{\text {ref }}, u_{\text {ref }}, l_{\text {ref }}$ and $p_{\text {ref }}$; for time, energy and temperature we take

$$
\begin{aligned}
t_{\mathrm{ref}} & =\frac{l_{\mathrm{ref}}}{u_{\mathrm{ref}}}, \\
e_{\mathrm{ref}} & =\frac{P_{\mathrm{ref}}}{\rho_{\mathrm{ref}}}, \\
T_{\mathrm{ref}} & =\frac{e_{\mathrm{ref}}}{c_{v}}=\frac{P_{\mathrm{ref}}}{\rho_{\mathrm{ref}} c_{v}} .
\end{aligned}
$$

Using the notation $a^{*}=a / a_{\text {ref }}$ for a generic variable $a$, we write the compressible Navier-Stokes 
equations (4) in non-dimensional form as

$$
\left\{\begin{array}{l}
\frac{\partial \rho^{*}}{\partial t^{*}}+\vec{\nabla}^{*} \cdot\left(\rho^{*} \vec{u}^{*}\right)=0 \\
\frac{\partial \rho^{*} \vec{u}^{*}}{\partial t^{*}}+\vec{\nabla}^{*} \cdot\left(\rho^{*} \vec{u}^{*} \otimes \vec{u}^{*}+\frac{1}{\gamma \mathrm{M}^{2}} p^{*} \underline{I}\right)=\frac{\vec{g}}{g} \frac{1}{\operatorname{Fr}} \rho^{*}+\frac{1}{\operatorname{Re}}\left(\vec{\nabla}^{*} \cdot \underline{\tau}^{*}\right) \\
\frac{\partial \rho^{*} e_{t}^{*}}{\partial t^{*}}+\vec{\nabla}^{*} \cdot\left(\left(\rho^{*} e_{t}^{*}+p^{*}\right) \vec{u}^{*}\right)=\frac{\gamma}{\operatorname{RePr}} \vec{\nabla}^{*} \cdot\left(\vec{\nabla}^{*} T^{*}\right)+\frac{\gamma \mathrm{M}^{2}}{\operatorname{Fr}} \frac{\vec{g}}{g} \cdot \rho^{*} \vec{u}^{*}+\frac{\gamma \mathrm{M}^{2}}{\operatorname{Re}} \vec{\nabla}^{*} \cdot\left(\underline{\tau}^{*} \cdot \vec{u}^{*}\right) \\
p^{*}=(\gamma-1) \rho^{*} T^{*}=(\gamma-1) \rho^{*}\left(e_{t}^{*}-\frac{\gamma \mathrm{M}^{2}}{2} \vec{u}^{*} \cdot \vec{u}^{*}\right)=(\gamma-1) \rho^{*} e^{*}
\end{array}\right.
$$

with

$$
\begin{aligned}
\underline{\tau}^{*}=\left(\vec{\nabla}^{*} \otimes \vec{u}^{*}+\right. & \left.\left(\vec{\nabla}^{*} \otimes \vec{u}^{*}\right)^{T}-\frac{2}{3}\left(\vec{\nabla}^{*} \cdot \vec{u}^{*}\right) \underline{I}\right), \\
\mathrm{M}^{2} & =\frac{u_{\mathrm{ref}}^{2} \rho_{\mathrm{ref}}}{\gamma P_{\mathrm{ref}}}=\frac{1}{\gamma \mathrm{Eu}}, \\
\operatorname{Re} & =\frac{u_{\mathrm{ref}} \rho_{\mathrm{ref}} l_{\mathrm{ref}}}{\mu}, \\
\operatorname{Pr} & =\frac{c_{p} \mu}{\lambda}, \\
\mathrm{Fr} & =\frac{u_{\mathrm{ref}}^{2}}{g l_{\mathrm{ref}}},
\end{aligned}
$$

where $\mathrm{M}$ is the Mach number, Eu the Euler number, Re the Reynolds number, Fr the Froude number and Pr the Prandtl number.

The equation expressing the conservation of internal energy (28) can be written in nondimensional form as

$$
\frac{\partial \rho^{*} e^{*}}{\partial t^{*}}+\vec{\nabla}^{*} \cdot\left(\rho^{*} \vec{u}^{*} e^{*}\right)+p^{*} \vec{\nabla}^{*} \cdot \vec{u}^{*}-\frac{\gamma \mathrm{M}^{2}}{\operatorname{Re}} \underline{\tau}^{*}: \vec{\nabla}^{*} \vec{u}^{*}-\frac{\gamma}{\operatorname{RePr}} \vec{\nabla}^{*} \cdot\left(\vec{\nabla}^{*} T^{*}\right)=0
$$

where

$$
e^{*}=e_{t}^{*}-\frac{\gamma \mathrm{M}^{2}}{2} \vec{u}^{*} \cdot \vec{u}^{*}=\frac{1}{\gamma-1} \frac{p^{*}}{\rho^{*}}=T^{*}
$$

The time-evolution equation for the temperature (29) can be written in non-dimensional form as

$$
\rho^{*}\left(\frac{\partial T^{*}}{\partial t^{*}}+\vec{u}^{*} \cdot \vec{\nabla}^{*} T^{*}\right)-\frac{1}{\gamma}\left(\frac{\partial p^{*}}{\partial t^{*}}+\vec{u}^{*} \cdot \vec{\nabla}^{*} p^{*}\right)-\frac{\mathrm{M}^{2}}{\operatorname{Re}} \tau^{*}: \vec{\nabla}^{*} \vec{u}^{*}-\frac{1}{\operatorname{RePr}} \vec{\nabla}^{*} \cdot\left(\vec{\nabla}^{*} T^{*}\right)=0 .
$$

The time-evolution equation for the velocity can be written in non-dimensional form as

$$
\rho^{*} \frac{\partial \vec{u}^{*}}{\partial t^{*}}+\rho^{*} \vec{u}^{*} \cdot \vec{\nabla}^{*} \vec{u}^{*}=-\frac{1}{\gamma \mathrm{M}^{2}} \vec{\nabla}^{*} p^{*}+\frac{\vec{g}}{g} \frac{1}{\operatorname{Fr}} \rho^{*}+\frac{1}{\operatorname{Re}}\left(\vec{\nabla}^{*} \cdot \underline{\tau}^{*}\right) .
$$




\subsection{Single time scale and single space scale asymptotic analysis}

Let us perform the single time scale and single space scale asymptotic analysis of the system involving the equation of conservation of internal energy (31), the time-evolution equation for the temperature (32) and the time-evolution equation for the velocity (33). In these equations, we insert the asymptotic expansion of the variables $\rho^{*}, \vec{u}^{*}, p^{*}$ and $T^{*}$ with respect to M. For the generic non-dimensional variable $a^{*}$ we can write

$$
a^{*}=a_{0}^{*}+\mathrm{M} a_{1}^{*}+\mathrm{M}^{2} a_{2}^{*}+\mathrm{O}\left(\mathrm{M}^{3}\right)
$$

Inserting these expansions in the time-evolution equation for the velocity (33) and equating the terms with identical power of $\mathrm{M}$, under the hypotheses

$$
1>>\frac{\gamma \mathrm{M}^{2}}{\mathrm{Fr}}=\frac{\rho_{\mathrm{ref}} g l_{\mathrm{ref}}}{p_{\mathrm{ref}}}
$$

and

$$
\frac{\mathrm{M}^{2}}{\mathrm{Re}}<<1
$$

we deduce that

$$
-\frac{1}{\gamma \mathrm{M}^{2}} \vec{\nabla}^{*} p_{0}^{*}=0
$$

and

$$
-\frac{1}{\gamma \mathrm{M}} \vec{\nabla}^{*} p_{1}^{*}=0
$$

i.e. $p_{0}^{*}$ and $p_{1}^{*}$ should be constant in space. It follows that

$$
\begin{aligned}
p^{*}\left(\vec{r}^{*}, t^{*}\right) & =\left(p_{0}^{*}\left(t^{*}\right)+\mathrm{M} p_{1}^{*}\left(t^{*}\right)\right)+\mathrm{M}^{2} p_{2}^{*}\left(\vec{r}^{*}, t^{*}\right)+\mathrm{O}\left(\mathrm{M}^{3}\right) \\
& =P^{*}\left(t^{*}\right)+p^{\prime *}\left(\vec{r}^{*}, t^{*}\right)
\end{aligned}
$$

with $p^{\prime *}\left(\vec{r}^{*}, t^{*}\right)=\mathrm{O}\left(\mathrm{M}^{2}\right)$. Equation (34) is the non-dimensional form of equation (5). Inserting expression (34) in the non-dimensional equation of state, we find that

$$
P^{*}\left(t^{*}\right)+\mathrm{O}\left(\mathrm{M}^{2}\right)=(\gamma-1) \rho^{*} T^{*}=(\gamma-1) \rho^{*} e^{*}
$$

which, neglecting the term $\mathrm{O}\left(\mathrm{M}^{2}\right)$ in the pressure asymptotic expansion, becomes

$$
P^{*}\left(t^{*}\right)=(\gamma-1) \rho^{*} T^{*}=(\gamma-1) \rho^{*} e^{*} .
$$

According to the (asymptotic) equation of state (36), the first term $P^{*}\left(t^{*}\right)$ in the RHS of equation (34) is called thermodynamic pressure. The second term $p^{\prime *}\left(\vec{r}^{*}, t^{*}\right)$ is called dynamic pressure; as already mentioned it is $\mathrm{O}\left(\mathrm{M}^{2}\right)$. Inserting expressions (34) and (35) in the equation of conservation of internal energy (31), we find that

$$
\begin{aligned}
& \frac{1}{\gamma-1} \frac{\partial}{\partial t^{*}}\left(P^{*}\left(t^{*}\right)+\mathrm{O}\left(\mathrm{M}^{2}\right)\right)+\frac{1}{\gamma-1} \vec{\nabla}^{*} \cdot\left(\left(P^{*}\left(t^{*}\right)+\mathrm{O}\left(\mathrm{M}^{2}\right)\right) \vec{u}^{*}\right)+\left(P^{*}\left(t^{*}\right)+\mathrm{O}\left(\mathrm{M}^{2}\right)\right) \vec{\nabla}^{*} \cdot \vec{u}^{*} \\
& -\frac{\gamma \mathrm{M}^{2}}{\operatorname{Re}} \underline{\tau}^{*}: \vec{\nabla}^{*} \vec{u}^{*}-\frac{\gamma}{\operatorname{RePr}} \vec{\nabla}^{*} \cdot\left(\vec{\nabla}^{*} T^{*}\right)=0
\end{aligned}
$$


which, neglecting the term $\mathrm{O}\left(\mathrm{M}^{2}\right)$ in the pressure asymptotic expansions and the viscous dissipation, gives

$$
\frac{1}{\gamma-1} \frac{\mathrm{d}}{\mathrm{d} t^{*}} P^{*}\left(t^{*}\right)+\frac{\gamma}{\gamma-1} P^{*}\left(t^{*}\right) \vec{\nabla}^{*} \cdot \vec{u}^{*}-\frac{\gamma}{\operatorname{RePr}} \vec{\nabla}^{*} \cdot\left(\vec{\nabla}^{*} T^{*}\right)=0 .
$$

This equation is the non-dimensional form of the first equation of (6).

Let us now insert expression (34) in the time-evolution equation for the temperature. We find that

$$
\begin{aligned}
& \rho^{*}\left(\frac{\partial T^{*}}{\partial t^{*}}+\vec{u}^{*} \cdot \vec{\nabla}^{*} T^{*}\right)-\frac{1}{\gamma}\left(\frac{\partial}{\partial t^{*}}\left(P^{*}\left(t^{*}\right)+\mathrm{O}\left(\mathrm{M}^{2}\right)\right)+\vec{u}^{*} \cdot \vec{\nabla}^{*}\left(P^{*}\left(t^{*}\right)+\mathrm{O}\left(\mathrm{M}^{2}\right)\right)\right) \\
& -\frac{\mathrm{M}^{2}}{\operatorname{Re}} \tau^{*}: \vec{\nabla}^{*} \overrightarrow{u^{*}}-\frac{1}{\operatorname{RePr}} \vec{\nabla}^{*} \cdot\left(\vec{\nabla}^{*} T^{*}\right)=0
\end{aligned}
$$

which, neglecting the term $\mathrm{O}\left(\mathrm{M}^{2}\right)$ in the pressure asymptotic expansions and the viscous dissipation, gives

$$
\rho^{*}\left(\frac{\partial T^{*}}{\partial t^{*}}+\vec{u}^{*} \cdot \vec{\nabla}^{*} T^{*}\right)-\frac{1}{\gamma} \frac{\mathrm{d}}{\mathrm{d} t^{*}} P^{*}\left(t^{*}\right)-\frac{1}{\operatorname{RePr}} \vec{\nabla}^{*} \cdot\left(\vec{\nabla}^{*} T^{*}\right)=0 .
$$

This equation is the non-dimensional form of the time-evolution equation for the temperature in (11). If we insert the equation of state (35) in this equation and we neglect the term $\mathrm{O}\left(\mathrm{M}^{2}\right)$ in the pressure asymptotic expansion, we obtain

$$
\frac{P^{*}}{(\gamma-1) T^{*}}\left(\frac{\partial T^{*}}{\partial t^{*}}+\vec{u}^{*} \cdot \vec{\nabla}^{*} T^{*}\right)-\frac{1}{\gamma} \frac{\mathrm{d}}{\mathrm{d} t^{*}} P^{*}\left(t^{*}\right)-\frac{1}{\operatorname{RePr}} \vec{\nabla}^{*} \cdot\left(\vec{\nabla}^{*} T^{*}\right)=0,
$$

which is the non-dimensional form of the time-evolution equation for the temperature in (6).

Finally, let us summarize the approximations thus introduced in the asymptotic models (6) and (11).

- In the asymptotic model (11), the first equation, the equation of conservation of mass, is not affected by any low Mach number approximation. We note that the time-evolution equation for the speed is not affected by the asymptotic analysis, in the sense that we can always subtract from the pressure a term which is constant in space [28, 34]. In the time-evolution equation for the temperature we neglect the term $\mathrm{O}\left(\mathrm{M}^{2}\right)$ in the pressure asymptotic expansion and the viscous dissipation. In all the equations, the computation of the density is performed via the (asymptotic) equation of state (36), which does not take into account the contribution of the dynamic pressure.

- In the asymptotic model (6), the equation of conservation of internal energy is obtained neglecting the contribution of the dynamic pressure in the internal energy and the viscous dissipation. In the time-evolution equation for the temperature, we neglect the term $\mathrm{O}\left(\mathrm{M}^{2}\right)$ in the pressure asymptotic expansion as well as the temperature variation due to the viscous dissipation. In all the equations, the density is replaced using the (asymptotic) equation of state (36), in which the contribution of the dynamic pressure is neglected.

In passing, we notice that in the literature there exist, for the Euler equations, asymptotic models which uses asymptotic analysis just to rearrange the terms in order to decide how 
to build the numerical method, without neglecting terms in the compressible Navier-Stokes equations (see for instance [34] and [24]). It would be interesting to extend such approaches to the Navier-Stokes equations and analyze them on this test case in terms of accuracy and CPU-time consumption.

\section{REFERENCES}

1. Barth TJ, Jespersen DC. The design and application of upwind schemes on unstructured meshes. AIAA Paper 89-0366, 1989

2. Brezzi F, Fortin M. Mixed and Hybrid Finite Element Methods. Springer-Verlag, 1991

3. Candel S. Mécanique des fluides. Dunod, 1995

4. Cast3M site. http://www-cast3m.cea.fr/cast3m/index.jsp [9 June 2006].

5. Chenoweth DR and Paolucci S. Natural convection in an enclosed vertical air layer with large horizontal temperature differences. Journal of Fluid Mechanics 1986; 169: 173-210.

6. Dhatt G, Touzot G. Une présentation de la méthode des éléments finis. Maloine S.A. Éditeur, 1981.

7. Elmo M, Cioni O. Low Mach number model for compressible flows and application to HTR. Nuclear Engineering and Design 2003; 222: 117-124.

8. Fröhlich J. Résolution numérique des équations de Navier-Stokes à faible nombre de Mach par une méthode spectrale. PhD Thesis. Université de Nice-Sophia Antipolis, 1990.

9. Gray D, Giorgini A. The validity of the Boussinesq approximation for liquids and gases. Heat and Mass Transfer 1976; 15: 545-551.

10. Gresho PM, Sani RL. Incompressible Flow and the Finite Element Method. John Wiley and Sons, 1998.

11. Kljenak I, Babi M, Mavko B, Bajsi I. Modeling of containment atmosphere mixing and stratification experiment using a CFD approach. Nuclear Engineering and Design 2006; 236: 1682-1692

12. Kloczko T. Développement d'une méthode implicite sans matrice pour la simulation 2D-3D des écoulements compressibles et faiblement compressibles en maillages non-structurés. PhD thesis. ENSAM Paris, 2006.

13. Le Quere P, Weisman C, Paillere H, Vierendeels J, Dick E, Becker R, Braack M, Locke, J. Modelling of natural convection flows with large temperature differences: a benchmark problem for low Mach number solvers. Part 1. Reference solutions. Mathematical Modelling and Numerical Analysis 2005. 39, 609-616

14. Luo H, Baum JD, Lohner R. Extension of Harten-Lax-van Leer Scheme for Flows at All Speeds. AIAA Journal 2005; 43 (6): 1160-1166.

15. Mathematical and Numerical aspects of Low Mach Number Flows June 21-25, 2004 / Porquerolles, France. http://www-sop.inria.fr/smash/LOMA/ [9 August 2006].

16. Meister A. Asymptotic single and multiple scale expansions in the low Mach number limit. SIAM J. Appl. Math. 1999; 60: 256-271.

17. Munz CD, Roller S, Klein R. Geratz KJ. The extension of incompressible flow solvers to the weakly compressible regime. Computer \& Fluids 2003; 32: 173-196.

18. Najm HM, Knio OM. Modeling Low Mach Number Reacting Flow with Detailed Chemistry and Transport. Journal of Scientific Computing 2005; 25: 263-287.

19. Noh WF. CEL : a time-dependent two-space dimensional coupled Eulerian-Lagrangian code. Methods in Computational Physics 1964; 3: 117-179.

20. Paillere H, Viozat C, Kumbaro A, Toumi I. Comparison of low Mach number models for natural convection. Heat and Mass Transfer 2000; 36: 567-573.

21. Paillere H, Le Quere P, Weisman C, Vierendeels J, Dick E, Braack M, Dabbene F, Beccantini A, Studer E, Kloczko, T. Modelling of natural convection flows with large temperature differences: a benchmark problem for low Mach number solvers. Part 2. Contributions. Mathematical Modelling and Numerical Analysis 2005. 39, 617-621

22. Paolucci S. On the filtering of sound from the Navier-Stokes equations. Technical Report SAND 82-8251, Sandia National Laboratories, Livermore, 1982

23. Pulliam TH, MacCormack RW, Venkateswaran S. Convergence characteristics of several approximate factorization methods. In ICNMDF98 $16^{\text {th }}$ International Conference on Numerical Methods in Fluid Dynamics; 1998.

24. Park JH, Munz CD. Multiple pressure variables methods for fluid flow at all Mach numbers. International Journal for Numerical Methods in Fluids 2005; 49: 905-931

25. Royl P, Rochholz H, Breitung W, Travis JR, Necker G. Analysis of steam and hydrogen distribution with PAR mitigation in NPP containments. Nuclear Engineering and Design 2000; 202: 231-248 
26. Saad Y. Iterative methods for sparse linear systems. Boston : PWS Pub.Co., 1996.

27. Saad Y, van der Vorst HA. Iterative solution of linear systems in the 20th century. Journal of Computational and Applied Mathematics 2000; 123: 1-33

28. Sesterhenn J, Müller B, Thomann H. On the Cancellation Problem in Calculating Compressible Low Mach Number Flows. Journal of Computational Physics 1999; 20: 597-615

29. Scheuerer M, Heitsch M, Menter F, Egorov, Y, Toth I, Bestion D, Pigny S, Paillere H, Martin A, Boucker M, Krepper E, Willemsen S, Muhlbauer P, Andreani M, Smith, B, Karlsson R, Henriksson, M, Hemstrom B, Karppinen I, Kimber, G. Evaluation of computational fluid dynamic methods for reactor safety analysis (ECORA). Nuclear Engineering and Design 2005; 235: 359-368

30. Siccama NB, Houkema M, Komen EMJ. CFD analyses of steam and hydrogen distribution in a nuclear power plant. In Use of Computational Fluid Dynamics Codes for Safety Analysis of Nuclear Reactor Systems. International Atomic Energy Agency, IAEA-TEDOC-1379, Vienna, Austria; 2003

31. Toro EF, Spruce M, Speares W. Restoration of the contact surface in the HLL Riemann Solver Shock Waves 1994; 4: 25-34

32. Turkel E. Preconditioned methods for solving the incompressible and low speed compressible equations. Journal of Computational Physics 1987; 72: 277-298.

33. Turkel E. Preconditioning techniques in computational fluid dynamics. Annual Review of Fluid Mechanics 1999; 31: 385-416.

34. van der Heul DR, Vuik C, Wesseling P. A conservative pressure-correction method for flow at all speeds. Computers \&S Fluids 2003; 32: 1113-1132.

35. Viozat C. Calcul d'écoulement stationnaire et instationnaire à petit nombre de Mach et en maillages étirés. PhD thesis. Université de Nice-Sophia Antipolis, 1998. 